\title{
Similarity between interval-valued fuzzy sets taking into account the width of the intervals and admissible orders
}

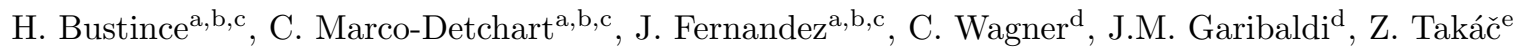 \\ ${ }^{a}$ Departamento de Estadistica, Informatica y Matematicas, Universidad Publica de Navarra, Campus de Arrosadia, 31006, \\ Pamplona, Spain \\ ${ }^{b}$ Institute of Smart Cities, Universidad Publica de Navarra, Campus de Arrosadia, 31006, Pamplona, Spain \\ ${ }^{c}$ Laboratory Navarrabiomed, Hospital Complex of Navarre (CHN), Universidad Publica de Navarra, IdiSNA, Irunlarrea 3. \\ 31008, Pamplona, Spain \\ ${ }^{d}$ School of Computer Science, University of Nottingham, Nottingham, United Kingdom \\ ${ }^{e}$ Institute of Information Engineering, Automation and Mathematics, Slovak University of Technology in Bratislava, \\ Radlinskeho 9, Bratislava, Slovakia
}

\begin{abstract}
In this work we study a new class of similarity measures between interval-valued fuzzy sets. The novelty of our approach lays, firstly, on the fact that we develop all the notions with respect to total orders of intervals; and secondly, on that we consider the width of intervals so that the uncertainty of the output is strongly related to the uncertainty of the input. For constructing the new interval-valued similarity, interval valued aggregation functions and interval-valued restricted equivalence functions which take into account the width of the intervals are needed, so we firstly study these functions, both in line with the two above stated features. Finally, we provide an illustrative example which makes use of an interval-valued similarity measure in stereo image matching and we show that the results obtained with the proposed interval-valued similarity measures improve numerically (according to the most widely used measures in the literature) the results obtained with interval valued similarity measures which do not consider the width of the intervals.
\end{abstract}

Keywords: Interval-valued fuzzy sets; Admissible order; Total order; Interval-valued similarity measure; Equivalence and restricted equivalence functions; Interval-valued aggregation function.

\section{Introduction}

In recent times, interval-valued fuzzy sets [12] are increasingly used in the same problems as standard type-1 fuzzy sets (from now on, simply referred to as fuzzy sets). This is due to the fact that, among other factors, they provide a way to represent the uncertainty inherent to the construction of an appropriate fuzzy set to represent a given setting. In this way, they may improve the numerical results of applications, as can be seen in $[1,2,3,5,6,7,17,18,28,36]$. Since many applications of fuzzy sets make use of similarity measures $[8,10,11,30]$ (see also the recent works $[15,16]$ ) in order to determine the degree of resemblance between fuzzy sets, interval-valued similarities have become also an object of interest for some authors $[19,25,31,42]$.

However, two observations can be stated regarding the recent literature about interval-valued similarities, and, more generally, about interval-valued fuzzy sets:

1. in most of the cases, only the partial order between intervals is considered;

2. the widths of the intervals are not considered.

Email addresses: bustince@unavarra.es (H. Bustince), cedric.marco@unavarra.es (C. Marco-Detchart), fcojavier.fernandez@unavarra.es (J. Fernandez), christian.wagner@nottingham.ac.uk (C. Wagner), jon.garibaldi@nottingham.ac.uk (J.M. Garibaldi), zdenko.takac@stuba.sk (Z. Takáč) 
In our opinion these two features are obstacles to further development of the theory and applications of interval-valued fuzzy sets.

Taking these factors into account, the objective of this paper is to construct similarity measures between interval-valued fuzzy sets in such a way that:

a) a total order for intervals (not only partial) is used;

b) the widths of intervals are considered.

With respect to objective (a), while in some applications it is not necessary for intervals to be comparable, in other cases (e.g. decision making or classification) in order to get a solution, it is important that any two intervals can be compared. Furthermore, it is desirable that some well-known notion which provides good results in the fuzzy setting and which involve the ordering of elements (such as OWA operators, Choquet integrals and so on) can be generalized in a natural way.

Regarding objective (b), we assume that the width of the membership interval of an element in a given set reflects the lack of knowledge of the precise membership degree of the element to the fuzzy set. In other words, we adopt an epistemic interpretation of the membership interval capturing incomplete information in respect to the actual degree of membership. Note that this interpretation is akin to that of a confidence interval and is in contrast to an alternative, ontic representation, where the interval itself represents the actual intervalvalued membership degree [21]. Thus, as we adopt an epistemic interpretation of the membership interval, we assume that there is one actual, real-valued membership degree of an element inside the membership interval of possible membership degrees, and consequently two elements with the same interval membership need not necessarily have the same (unknown) actual real-valued membership degree.

To achieve objectives (a) and (b), we first introduce new definitions of interval-valued aggregation functions and interval-valued restricted equivalence functions, both in line with the observations stated above. It is worth pointing out that this is the first time in the literature that interval functions are studied according to these observations, and we assume that the approach can be utilized in a wide range of problems featuring intervals in the future.

Ideally, the definition of width-preserving interval-valued restricted equivalence functions (IVREF) would have to take into account the width of the inputs in every case. In other words, the width of the output interval should always be related by some axiom to the width of the input intervals, and not only when the input intervals have the same length. In this way, the epistemic interpretation would be fully preserved. However, it is not clear which this relation exactly should be and furthermore, how such IVREF could be constructed taking into account the complexity of the analysis and the construction when general admissible orders are involved. For this reason, the proposed set of axioms provides a first step in the desired direction.

To show the validity of our approach, we present an application using an expression of the proposed interval-valued similarity measure taking into account the width of the intervals which provides better results than other methods that can be found in the literature. In particular, we describe the application of our similarity in stereo image matching and show that it outperforms the classical methods that make use of interval-valued fuzzy sets but do not take into account the width of the membership intervals.

The paper is organized as follows. We start with some preliminaries, then we study the concepts of interval-valued restricted equivalence functions and interval-valued aggregation functions preserving the widths of intervals. In Section 4, we introduce the definition of width-based interval-valued similarity measures and study different construction methods. In Section 5, we present an illustrative example of application of width-based interval-valued similarity measures in stereo image matching. We finish with some conclusions and references.

\section{Preliminaries}

In this section, we introduce several well known notions and results which are necessary for our subsequent developments.

We start recalling the idea of aggregation function. For more details, see [32]. 
Definition 2.1. An aggregation function is a non-decreasing function $M:[0,1]^{n} \rightarrow[0,1]$ with $M(0, \ldots, 0)=$ 0 and $M(1, \ldots, 1)=1$.

An aggregation function $M:[0,1]^{n} \rightarrow[0,1]$ is called idempotent if $M(x, \ldots, x)=x$ for every $x \in[0,1]$, and it is called symmetric if $M\left(x_{1}, \ldots, x_{n}\right)=M\left(x_{\sigma(1)}, \ldots, x_{\sigma(n)}\right)$ for every $x_{1}, \ldots, x_{n} \in[0,1]$ and every permutation $\sigma:\{1, \ldots, n\} \rightarrow\{1, \ldots, n\}$.

Among the most relevant classes of aggregation functions we can mention the following.

Definition 2.2. A t-norm is a symmetric aggregation function $T:[0,1]^{2} \rightarrow[0,1]$ such that $T(x, 1)=x$ and $T(T(x, y), z)=T(x, T(y, z))$ for every $x, y, z \in[0,1]$.

Definition 2.3. A t-conorm is a symmetric aggregation function $S:[0,1]^{2} \rightarrow[0,1]$ such that $S(x, 0)=x$ and $S(S(x, y), z)=S(x, S(y, z))$ for every $x, y, z \in[0,1]$.

Among the most significant t-norms we can mention the minimum or the product, whereas among the most relevant t-conorms we can cite the maximum or the probabilistic sum $S_{P}(x, y)=x+y-x y$ [32].

In this work we are going to deal with closed subintervals of the unit interval. We denote by $L([0,1])$ the set of closed subintervals of the unit interval, that is:

$$
L([0,1])=\{[\underline{X}, \bar{X}] \mid 0 \leq \underline{X} \leq \bar{X} \leq 1\} .
$$

We use capital letters to denote elements in $L([0,1])$. The width of the interval $X \in L([0,1])$ is denoted by $w(X)$, where $w(X)=\bar{X}-\underline{X}$. An interval function $f:(L([0,1]))^{n} \rightarrow L([0,1])$ is called width-preserving (or $w$-preserving, for simplicity) if, for any $X_{1}, \ldots, X_{n} \in L([0,1])$ such that $w\left(X_{1}\right)=\ldots=w\left(X_{n}\right)$, it holds that $w\left(f\left(X_{1}, \ldots, X_{n}\right)\right)=w\left(X_{1}\right)$.

We work on a finite universe $U=\left\{u_{1}, \ldots, u_{n}\right\}$. An interval-valued fuzzy set (IVFS) on the universe $U$ is a mapping $A: U \rightarrow L([0,1])$. The class of all fuzzy sets in $U$ is denoted by $F S(U)$ and the class of all interval-valued fuzzy sets in $U$ by $I V F S(U)$. Given $A \in I V F S(U)$, its entropy is defined as

$$
\epsilon(A)=\sum_{i=1}^{n}\left(\bar{A}\left(u_{i}\right)-\underline{A}\left(u_{i}\right)\right) .
$$

Note that this entropy measures how far from fuzzy sets a given IVFS is, see [9] for more details. In this sense, although we have kept the original name of entropy which appears in [9], it is a non-specificity index for interval membership grades which differs from the usual fuzzy entropy related to crispness of fuzzy sets.

Another key notion in this work is that of order relation. Recall that an order relation on $L([0,1])$ is a binary relation $\leq$ on $L([0,1])$ which is reflexive, symmetric and transitive. An order relation on $L([0,1])$ is called total or linear if any two elements of $L([0,1])$ are comparable, i.e., if for every $X, Y \in L([0,1]), X \leq Y$ or $Y \leq X$. An order relation on $L([0,1])$ is partial if it is not total.

Although many different orders can be provided in $L([0,1])$, we are interested in the lattice extension of the ordering in $[0,1]$, that we will denote by $\precsim_{L}$ and which is the partial order given by:

$$
[\underline{X}, \bar{X}] \precsim_{L}[\underline{Y}, \bar{Y}] \quad \text { if } \quad \underline{X} \leq \underline{Y} \text { and } \bar{X} \leq \bar{Y} .
$$

It is worth mentioning that with this order, any two degenerate intervals (i.e., intervals of width 0) are comparable. In particular, this implies that if the interval-valued fuzzy sets we are going to deal with are in fact fuzzy sets (that is, if all the membership intervals have width 0), the order between the intervalvalued fuzzy sets is the same as the order between the fuzzy sets. In other words, if we use this order (or any extension of it), we can extend algorithms in the fuzzy setting which make use of the order in a straightforward way. This is the order relation most widely used in the literature [14].

We denote by $\leq_{L}$ any order on $L\left([0,1]\right.$ ) (which can be partial or total) with $0_{L}=[0,0]$ as its minimal element (that is, $0_{L} \leq_{L} X$ for all $\left.X \in L([0,1])\right)$ and $1_{L}=[1,1]$ as its maximal element (that is, $X \leq_{L} 1_{L}$ for all $X \in L([0,1]))$. To denote a total order on $L([0,1])$ with the same minimal and maximal elements, we use the notation $\leq_{T L}$. 
Example 2.4. (i) A total order on $L([0,1])$ is, for example, the $\mathrm{Xu}$ and Yager's order $\leq_{X Y}$ (see $[41]$ ):

$$
[\underline{X}, \bar{X}] \leq_{X Y}[\underline{Y}, \bar{Y}] \quad \text { if } \quad\left\{\begin{array}{l}
\underline{X}+\bar{X}<\underline{Y}+\bar{Y} \text { or } \\
\underline{X}+\bar{X}=\underline{Y}+\bar{Y} \text { and } \bar{X}-\underline{X} \leq \bar{Y}-\underline{Y} .
\end{array}\right.
$$

This definition of Xu and Yager's order was originally provided for Atanassov intuitionistic fuzzy pairs [41].

(ii) Another example of total order is provided by the lexicographical order with respect to the first variable, $\leq_{l e x 1}$, and with respect to the second variable, $\leq_{l e x 2}$, which are defined, respectively, by:

$$
\begin{aligned}
& {[\underline{X}, \bar{X}] \leq_{l e x 1}[\underline{Y}, \bar{Y}] \quad \text { if } \quad\left\{\begin{array}{l}
\underline{X}<\underline{Y} \text { or } \\
\underline{X}=\underline{Y} \text { and } \bar{X} \leq \bar{Y} .
\end{array}\right.} \\
& {[\underline{X}, \bar{X}] \leq_{l e x 2}[\underline{Y}, \bar{Y}] \quad \text { if } \quad\left\{\begin{array}{l}
\bar{X}<\bar{Y} \text { or } \\
\bar{X}=\bar{Y} \text { and } \underline{X} \leq \underline{Y} .
\end{array}\right.}
\end{aligned}
$$

Regarding total orders on $L([0,1])$, we are going to consider the so-called admissible orders, whose definition we recall now.

Definition 2.5. [13] An admissible order on $L([0,1])$ is a total order $\leq_{T L}$ on $L([0,1])$ such that it refines the partial order $\precsim_{L}$, that is, for every $X, Y \in L([0,1])$, if $X \precsim_{L} Y$ then $X \leq_{T L} Y$.

An interesting feature of admissible orders is that they can be built using aggregation functions, as stated in the following result.

Proposition 2.6. ([13]) Let $M_{1}, M_{2}:[0,1]^{2} \rightarrow[0,1]$ be two aggregation functions such that for all $X, Y \in$ $L([0,1])$, the equalities $M_{1}(\underline{X}, \bar{X})=M_{1}(\underline{Y}, \bar{Y})$ and $M_{2}(\underline{X}, \bar{X})=M_{2}(\underline{Y}, \bar{Y})$ can only hold simultaneously if $X=Y$. The order $\leq_{M_{1}, M_{2}}$ on $L([0,1])$ given by

$$
X \leq_{M_{1}, M_{2}} Y \quad \text { if } \quad\left\{\begin{array}{l}
M_{1}(\underline{X}, \bar{X})<M_{1}(\underline{Y}, \bar{Y}) \text { or } \\
M_{1}(\underline{X}, \bar{X})=M_{1}(\underline{Y}, \bar{Y}) \text { and } M_{2}(\underline{X}, \bar{X}) \leq M_{2}(\underline{Y}, \bar{Y})
\end{array}\right.
$$

is an admissible order on $L([0,1])$.

Example 2.7. (i) $\mathrm{Xu}$ and Yager's order is an example of admissible order with $M_{1}(x, y)=\frac{x+y}{2}$ and $M_{2}(x, y)=y$.

(ii) The lexicographical orders $\leq_{l e x 1}\left(\leq_{l e x 2}\right)$ are also examples of admissible orders with $M_{1}(x, y)=x$ $\left(M_{1}(x, y)=y\right)$ and $M_{2}(x, y)=y\left(M_{2}(x, y)=x\right)$.

(iii) More generally, if, for $\alpha \in[0,1]$ we define the aggregation function

$$
K_{\alpha}(x, y)=(1-\alpha) x+\alpha y
$$

then, for $\alpha, \beta \in[0,1]$ with $\alpha \neq \beta$, we can obtain an admissible order $\leq_{\alpha, \beta}$ just taking $M_{1}(x, y)=$ $K_{\alpha}(x, y)$ and $M_{2}(x, y)=K_{\beta}(x, y)$. Observe that this operator $K_{\alpha}$ corresponds to Hurwicz's criterion [27] for balancing pessimism and optimism under uncertainty. See [13] for more details.

\subsection{Interval-valued aggregation functions with respect to a partial order}

The definition of aggregation function has been extended to the interval-valued setting with respect to the order $\precsim_{L}$ in a straightforward way [29].

Definition 2.8. Let $n \geq 2$. An ( $n$-dimensional) interval-valued (IV) aggregation function in $L([0,1])$ with respect to $\precsim_{L}$ is a mapping $M_{I V}:(L([0,1]))^{n} \rightarrow L([0,1])$ which verifies: 
(i) $M_{I V}\left(0_{L}, \cdots, 0_{L}\right)=0_{L}$.

(ii) $M_{I V}\left(1_{L}, \cdots, 1_{L}\right)=1_{L}$.

(iii) $M_{I V}$ is a non-decreasing function with respect to $\precsim_{L}$.

Remark 2.9. Note that this definition does not fully recover the usefulness of the usual definition of aggregation functions in the real setting (defined with respect to a total order) since there may exist intervals which are not comparable by means of the order $\precsim_{L}$, so the full meaning of monotonicity is lost.

It is quite easy to get IV aggregation functions in the sense of Def. 2.8, as the following examples show.

Example 2.10. ([34]) If $A:[0,1]^{2} \rightarrow[0,1]$ is an aggregation function, then the function $M_{A}: L([0,1])^{2} \rightarrow$ $L([0,1])$ given by

$$
M_{A}([\underline{X}, \bar{X}],[\underline{Y}, \bar{Y}])=[A(\underline{X}, \underline{Y}), A(\bar{X}, \bar{Y})],
$$

is an IV aggregation function in $L([0,1])$ with respect to the order $\precsim_{L}$.

Moreover, if $A, B:[0,1]^{2} \rightarrow[0,1]$ are two aggregation functions such that $A(x, y) \leq B(x, y)$ for each $x, y \in[0,1]$, then

$$
M_{A, B}([\underline{X}, \bar{X}],[\underline{Y}, \bar{Y}])=[A(\underline{X}, \underline{Y}), B(\bar{X}, \bar{Y})],
$$

is an IV aggregation function in $L([0,1])$ with respect to the order $\precsim_{L}$.

Example 2.11. The following functions are IV aggregation functions in $L([0,1])$ with respect to the order $\precsim L$.

- $M_{I V}([\underline{X}, \bar{X}],[\underline{Y}, \bar{Y}])=\left[(\underline{X Y})^{2},(\overline{X Y})^{2}\right]$,

- $M_{I V}([\underline{X}, \bar{X}],[\underline{Y}, \bar{Y}])=\left[\underline{X Y^{1 / 2}},(\bar{X}+\bar{Y}) / 2\right]$.

\subsection{Restricted equivalence functions}

Comparison measures between fuzzy sets can be built using the notion of restricted equivalence functions. We recall now this notion. For more details, see [11].

Definition 2.12. A function $R:[0,1]^{2} \rightarrow[0,1]$ is called a restricted equivalence function (REF) if it satisfies:

1. $R(x, y)=0$ if and only if $\{x, y\}=\{0,1\}$ (i.e., if and only if $|x-y|=1$ );

2. $R(x, y)=1$ if and only if $x=y$;

3. $R(x, y)=R(y, x)$ for all $x, y \in[0,1]$;

4. If $x \leq y \leq z$, then $R(x, z) \leq R(x, y)$ and $R(x, z) \leq R(y, z)$ for all $x, y, z \in[0,1]$.

Example 2.13. For any $p \in] 0, \infty\left[\right.$, the function $R^{p}(x, y)=1-|x-y|^{p}$ is a REF.

Remark 2.14. It is worth mentioning that, for some of our developments, we can consider the weaker condition

$$
\text { 2'. } R(x, x)=1 \text { for every } x \in[0,1]
$$

instead of the stronger condition 2. in Definition 2.12. With this condition, we are recovering equivalence functions, see [23]. 


\section{Width-preserving interval valued restricted equivalence functions}

In this section, we propose a new definition of restricted equivalence functions (REF) in the intervalvalued setting which takes into account the width of the inputs.

Definition 3.1. Let $\leq_{L}$ be an order on $L([0,1])$. An interval-valued restricted equivalence function w.r.t. the order $\leq_{L}$ is a function $R_{I V}: L([0,1])^{2} \rightarrow L([0,1])$ such that:

1. $R_{I V}(X, Y)=0_{L}$ if and only if $\{X, Y\}=\left\{0_{L}, 1_{L}\right\}$;

2. $R_{I V}(X, X)=[1-w(X), 1]$ for all $X \in L([0,1])$;

3. $R_{I V}(X, Y)=R_{I V}(Y, X)$ for all $X, Y \in L([0,1])$;

4. If $X, Y, Z \in L([0,1])$ are such that $X \leq_{L} Y \leq_{L} Z$ and $w(X)=w(Y)=w(Z)$, then $R_{I V}(X, Z) \leq_{L}$ $R_{I V}(X, Y)$ and $R_{I V}(X, Z) \leq_{L} R_{I V}(Y, Z)$.

\section{Justification of the axioms}

1. Axiom 1. recovers the property required in the definition of REF in the real-valued setting [11].

2. The main difference with respect to the definition of REFs in the fuzzy setting arises in axiom 2. Note that we consider that the width of the membership interval of an element in a given set is a measure of the lack of knowledge of the precise (real-valued) membership degree of that element, and it is assumed that the exact membership value is a value inside the membership interval. Thus, if two elements have the same interval memberships, this does not mean that their corresponding/underlying real-valued degrees of membership are the same. Hence it is natural to expect that we can not get less uncertainty when comparing them.

3. Symmetry is a natural requirement also demanded in the real-valued setting.

4. Regarding axiom 4., observe that in the real-valued case, a total order (i.e., the usual order between real numbers) is used, and hence any two valued obtained by means of a REF can be compared. If we consider that $\leq_{L}$ is a total order, we are also able to compare any two intervals that are obtained as the result of an interval-valued restricted equivalence function w.r.t. the order $\leq_{L}$. Nevertheless, this axiom is more flexible, since also partial orders can be considered. By imposing the restriction that $w(X)=w(Y)=w(Z)$, we are recovering the condition demanded in the real case, since if $X, Y, Z$ are intervals which consist of a single point, it follows that $w(X)=w(Y)=w(Z)=0$.

Remark 3.2. As noted in the introduction, this definition does not take into account the width of the input intervals in every case, but we consider it to be a first step in this direction.

Example 3.3. (i) A natural example of width preserving interval-valued restricted equivalence functions is the following:

$$
R_{\text {epis }}(X, Y)=\{1-|x-y| \mid x \in X, y \in Y\},
$$

which exactly reflects the epistemic nature of $X$ and $Y$, and is the interval extension of $1-|x-y|$. Note that, even if this function fulfills the four axioms in Def. 2.12, it does not take into account the width of the inputs. Note that $R_{\text {epis }}$ can also be expressed in terms of the endpoints of the intervals:

$$
R_{\text {epis }}(X, Y)=[1-\max (\bar{X}-\underline{Y}, \bar{Y}-\underline{X}), 1-\max (0, \max (\underline{X}, \underline{Y})-\min (\bar{X}, \bar{Y}))] .
$$

Furthermore, observe that in this case, if we take two intervals of the same width, as, for instance, $X=[0.5,0.7]$ and $Y=[0.6,0.8]$, the result $R_{\text {epis }}(X, Y)=[0.7,1]$ does not have the same width as the inputs. 
(ii) However, it is possible to provide other examples of width preserving interval-valued restricted equivalence functions such that, if the inputs have the same width, the output also has that same width. For instance, the function $R_{I V}: L([0,1])^{2} \rightarrow L([0,1])$ defined as:

$$
R_{I V}(X, Y)=\left[\max \left(0,1-\left|K_{\alpha}(X)-K_{\alpha}(Y)\right|-\frac{1}{2}(w(X)+w(Y))\right), \max \left(1-\left|K_{\alpha}(X)-K_{\alpha}(Y)\right|, \frac{1}{2}(w(X)+w(Y))\right)\right]
$$

is, for every $\alpha \in] 0,1]$, an example of IVREF w.r.t. any admissible order.

Note that in a sense this second example can be considered an approach similar to the $R_{\text {epis }}$. We are taking into account specific points in the interval by means of $K_{\alpha}$ operators (without the need for bounds, as is the case for $\left.R_{\text {epis }}\right)$. In particular, note that $R_{\text {epis }}$ can be written as:

$$
R_{\text {epis }}(X, Y)=\left[\min _{\alpha, \beta \in[0,1]}\left(1-\left|K_{\alpha}(X)-K_{\beta}(Y)\right|\right), \max _{\alpha, \beta \in[0,1]}\left(1-\left|K_{\alpha}(X)-K_{\beta}(Y)\right|\right)\right]
$$

but we can replace it by the following equivalent expression which depends only on $\alpha$ :

$$
R_{\text {epis }}(X, Y)=\left[\min _{\alpha \in[0,1]}\left(1-\left|K_{\alpha}(X)-K_{1-\alpha}(Y)\right|\right), \max _{\alpha \in[0,1]}\left(1-\left|K_{\alpha}(X)-K_{1-\alpha}(Y)\right|\right)\right] .
$$

Observe that $R_{I V}$, which depends on the parameter $\alpha$, is based on a similar idea as $R_{\text {epis }}$ (in the sense that they both generalize $1-|x-y|$ to intervals) with the distinction that it is constructed directly in line with the intuition behind the notion of $K_{\alpha, \beta}$ order, i.e., the choice of $\alpha$ naturally depends on the considered order. Moreover, $R_{I V}$ also takes into account the width of the inputs intervals whereas $R_{\text {epis }}$ does not.

At the same time, if we take $\alpha=0.5$ and the intervals $X=[0.3,0.7]$ and $Y=[0.5,0.7]$, we obtain IVREF equal to $[0.6,0.9]$, - even though it is possible that the 'real-value' of both sets is exactly the same (0.7). On the other hand, if we use $R_{\text {epis }}$, we see that the IVREF is equal to $[0.4,1]$, so we would recover the value 1.

Next we give a result regarding a monotonicity with respect to the widths of the inputs.

Proposition 3.4. Let $X, Y \in L([0,1])$. If $w(X)<w(Y)$, then, for any admissible order $\leq_{T L}$, it follows that

$$
R_{I V}(Y, Y) \leq_{T L} R_{I V}(X, X)
$$

Proof. It follows straightforwardly from Definition 3.1.

Let us consider now the construction of examples of width-preserving IVREFs with respect to an admissible order. First of all, observe that if we consider and expression of the type $R_{I V}(X, Y)=\{R E F(x, y) \mid$ $x \in X$ and $y \in Y\}$, as the natural interval-valued extension of a real-valued REF function, $R_{I V}$ is a widthpreserving IVREF with respect to an admissible order only if the second axiom in Def. 3.1 holds, and this happens if and only if $\operatorname{REF}(x, y)=1-|x-y|$. This means that in order to get general construction methods of examples that can be useful for applications, we need to find new construction methods. Furthermore, and in order to build width-preserving similarity functions, we are interested in IVREFs which preserve the width of the input intervals if they are the same.

Our first step is the following lemma, which recovers a feature of admissible orders.

Lemma 3.5. Let $X, Y \in L([0,1])$ be intervals such that $w(X)=w(Y)$. Then

$$
X \precsim_{L} Y \text { if and only if } X \leq_{T L} Y
$$

for any admissible order $\leq_{T L}$.

Proof. The proof follows from the observation that intervals with the same width are always comparable by the partial order $\precsim_{L}$ and admissible orders refine the partial order $\precsim_{L}$. 
Now we discuss a procedure to build IVREFs which preserve the width of input intervals, and which is based on the use of $K_{\alpha}$ operators. Note that these operators can be viewed as choosing one representative point inside the intervals. Thus, in order to ensure that the axioms in the definition are fulfilled, we also must take into account the widths of the input intervals.

Theorem 3.6. Let $\alpha \in] 0,1\left[\right.$, let $M:[0,1]^{2} \rightarrow[0,1]$ be an idempotent symmetric aggregation function and let $R:[0,1]^{2} \rightarrow[0,1]$ be a restricted equivalence function. Then, the function $R_{I V}: L([0,1])^{2} \rightarrow L([0,1])$ given by

$$
R_{I V}(X, Y)=\left[\max \left(0, R\left(K_{\alpha}(X), K_{\alpha}(Y)\right)-M(w(X), w(Y))\right), \max \left(R\left(K_{\alpha}(X), K_{\alpha}(Y)\right), M(w(X), w(Y))\right)\right]
$$

is an IV restricted equivalence function w.r.t. any admissible order $\leq_{T L}$. Moreover, $R_{I V}$ is w-preserving.

Proof. For simplicity we write $\mathcal{R}$ instead of $R\left(K_{\alpha}(X), K_{\alpha}(Y)\right)$, and $\mathcal{M}$ instead of $M(w(X), w(Y))$. Then $(3)$ can be simplified:

$$
R_{I V}(X, Y)=[\max (0, \mathcal{R}-\mathcal{M}), \max (\mathcal{R}, \mathcal{M})]= \begin{cases}{[\mathcal{R}-\mathcal{M}, \mathcal{R}],} & \text { if } \mathcal{R} \geq \mathcal{M} \\ {[0, \mathcal{M}],} & \text { otherwise }\end{cases}
$$

By (4) it is clear that $R_{I V}$ is well-defined.

Observe that $R_{I V}(X, Y)=0_{L}$ if and only if $\mathcal{R}=0$ and $\mathcal{M}=0$. The former holds if and only if $\left\{K_{\alpha}(X), K_{\alpha}(Y)\right\}=\{0,1\}$, which may happen if and only if $\{X, Y\}=\left\{0_{L}, 1_{L}\right\}$. So it follows that $w(X)=w(Y)=0$ and we get the first condition in Definition 3.1.

The second condition in Definition 3.1 follows from the observations: $R\left(K_{\alpha}(X), K_{\alpha}(X)\right)=1$ and $M(w(X), w(X))=w(X)$.

Symmetry of $R_{I V}$ directly follows from the symmetry of $R$ and $M$.

The fulfillment of the fourth condition in Definition 3.1 w.r.t. any admissible order follows from the monotonicity of $R$, Lemma 3.5, after observing that, if $X \leq_{T L} Y \leq_{T L} Z$ and $w(X)=w(Y)=w(Z)$, then $K_{\alpha}(X) \leq K_{\alpha}(Y) \leq K_{\alpha}(Z)$.

Finally, the fact that $R_{I V}$ is $w$-preserving directly follows from Equation (4) and idempotency of $M$.

We can use any REF $R$ and any idempotent symmetric aggregation function $M$ in Equation (3) to build an IVREF. Equation (3) can be simplified if some additional assumptions on $R$ and $M$ are imposed.

Corollary 3.7. Let $\alpha \in] 0,1\left[\right.$, let $M:[0,1]^{2} \rightarrow[0,1]$ be an idempotent symmetric aggregation function such that $M(x, y) \leq \min ((1-\alpha) x+\alpha y, \alpha x+(1-\alpha) y)$ for all $x, y \in[0,1]$, and let $R:[0,1]^{2} \rightarrow[0,1]$ be a restricted equivalence function such that $R(x, y) \geq 1-|x-y|$ for all $x, y \in[0,1]$. Then, the function $R_{I V}: L([0,1])^{2} \rightarrow L([0,1])$ given by

$$
R_{I V}(X, Y)=\left[R\left(K_{\alpha}(X), K_{\alpha}(Y)\right)-M(w(X), w(Y)), R\left(K_{\alpha}(X), K_{\alpha}(Y)\right)\right]
$$

is an IV restricted equivalence function w.r.t. any admissible order $\leq_{T L}$. Moreover, $R_{I V}$ is w-preserving.

Proof. We only need to prove that $R\left(K_{\alpha}(X), K_{\alpha}(Y)\right) \geq M(w(X), w(Y))$ for all $X, Y \in L([0,1])$, since in that case Equation (5) is a special case of Equation (3). Due to the assumptions on $M$ and $R$, it is enough to show that

$$
1-\left|K_{\alpha}(X)-K_{\alpha}(Y)\right| \geq \min ((1-\alpha) w(X)+\alpha w(Y), \alpha w(X)+(1-\alpha) w(Y)) .
$$

Assume that $K_{\alpha}(X) \geq K_{\alpha}(Y)$. Then

$$
1-\left|K_{\alpha}(X)-K_{\alpha}(Y)\right|=1-(1-\alpha) \underline{X}-\alpha \bar{X}+(1-\alpha) \underline{Y}+\alpha \bar{Y}
$$


and since

$$
1 \geq \bar{X}-\underline{Y}=(1-\alpha)(\bar{X}-\underline{Y})+\alpha(\bar{X}-\underline{Y})=(1-\alpha)(\bar{X}-\underline{X}+\underline{X}-\underline{Y})+\alpha(\bar{X}-\bar{Y}+\bar{Y}-\underline{Y}),
$$

we have

$$
1-(1-\alpha) \underline{X}-\alpha \bar{X}+(1-\alpha) \underline{Y}+\alpha \bar{Y} \geq(1-\alpha)(\bar{X}-\underline{X})+\alpha(\bar{Y}-\underline{Y})
$$

hence (6) is satisfied.

Now assume that $K_{\alpha}(X)<K_{\alpha}(Y)$. Then

$$
1-\left|K_{\alpha}(X)-K_{\alpha}(Y)\right|=1+(1-\alpha) \underline{X}+\alpha \bar{X}-(1-\alpha) \underline{Y}-\alpha \bar{Y}
$$

and since

$$
1 \geq \bar{Y}-\underline{X}=(1-\alpha)(\bar{Y}-\underline{X})+\alpha(\bar{Y}-\underline{X})=(1-\alpha)(\bar{Y}-\underline{Y}+\underline{Y}-\underline{X})+\alpha(\bar{Y}-\bar{X}+\bar{X}-\underline{X}),
$$

we have

$$
1+(1-\alpha) \underline{X}+\alpha \bar{X}-(1-\alpha) \underline{Y}-\alpha \bar{Y} \geq(1-\alpha)(\bar{Y}-\underline{Y})+\alpha(\bar{X}-\underline{X}),
$$

hence (6) is satisfied.

Remark 3.8. Note that in the previous Corollary we are considering REF functions $R$ which are greater than or equal to $1-|x-y|$, so in general, their interval extensions

$$
R_{I V}(X, Y)=\{R(x, y) \mid x \in X \text { and } y \in Y\}
$$

need not satisfy the axioms about width in Def. 3.1. However, we can make use of them to build new examples of width-preserving IVREFs, see also Ex. 3.11 below.

As a consequence, we show in the next Corollary that a width-preserving IVREF built as in Corollary 3.7 does not in fact decrease the width of the input intervals, even if the latter are different to each other, and the width of the resulting interval depends parametrically on $\alpha$. Furthermore, if $M=$ max, then the width of the output interval equals the maximum of the widths of the input intervals. Note that in the case of $R_{\text {epis }}$ this is not the case as, for instance, $R_{\text {epis }}([0.1,0.9],[0.3,0.5])=[0.4,1]$.

Corollary 3.9. Consider the interval-valued restricted equivalence function $R_{I V}$ constructed in Corollary 3.7. Then, for all $X, Y \in L([0,1])$, it holds that

$$
\min (w(X), w(Y)) \leq w\left(R_{I V}(X, Y)\right)=M(w(X), w(Y)) \leq \min ((1-\alpha) w(X)+\alpha w(Y), \alpha w(X)+(1-\alpha) w(Y)) .
$$

Proof. The first inequality follows from the fact that an idempotent aggregation function is always greater than or equal to the minimum and the second inequality follows from the property of $M$ assumed in Corollary 3.7.

We can also prove the following result.

Lemma 3.10. If $M_{1}, M_{2}:[0,1]^{2} \rightarrow[0,1]$ are idempotent symmetric aggregation functions, then the function $M:[0,1]^{2} \rightarrow[0,1]$ given by

$$
M(x, y)=\min \left(M_{1}(x, y), M_{2}(x, y)\right),
$$

for all $x, y \in[0,1]$, is an idempotent symmetric aggregation function.

Proof. The proof is straightforward. 
Example 3.11. Let us consider the construction of IVREF given by Corollary 3.7. Observe that the REF $R^{p}$ defined in Example 2.13 satisfies assumption of the corollary: $R^{p}(x, y) \geq R^{1}=1-|x-y|$ for all $x, y \in[0,1]$ if and only if $p \in[1, \infty[$.

(i) If we take $\alpha=1 / 2$ and $M(x, y)=\frac{x+y}{2}$ we get a class of IVREFs w.r.t. any admissible order:

$$
R_{I V}^{p}(X, Y)=\left[R^{p}\left(\frac{\underline{X}+\bar{X}}{2}, \frac{\underline{Y}+\bar{Y}}{2}\right)-\frac{w(X)+w(Y)}{2}, R^{p}\left(\frac{\underline{X}+\bar{X}}{2}, \frac{\underline{Y}+\bar{Y}}{2}\right)\right]
$$

for $p \in[1, \infty[$.

(ii) If we take $M(x, y)=\min (x, y)$, a class of IVREFs w.r.t. any admissible order arises:

$$
R_{I V}^{p, \alpha}(X, Y)=\left[R^{p}\left(K_{\alpha}(X), K_{\alpha}(Y)\right)-\min (w(X), w(Y)), R^{p}\left(K_{\alpha}(X), K_{\alpha}(Y)\right)\right]
$$

for $p \in[1, \infty[$ and $\alpha \in] 0,1[$.

(iii) Let $\alpha \in[0,1]$, it is easy to see that

$$
\min ((1-\beta) x+\beta y, \beta x+(1-\beta) y) \leq \min ((1-\alpha) x+\alpha y, \alpha x+(1-\alpha) y)
$$

for all $\beta \in[0,1]$ such that $\max (\beta, 1-\beta) \geq \max (\alpha, 1-\alpha)$. Hence, we get a more general class of IVREFs w.r.t. any admissible order than that in item (ii), if we take $\alpha \in] 0,1[$ and

$$
M(x, y)=\min ((1-\beta) x+\beta y, \beta x+(1-\beta) y)
$$

for $\beta \in[\max (\alpha, 1-\alpha), 1]$ (or equivalently for $\beta \in[0, \min (\alpha, 1-\alpha)]$ ):

$$
\begin{aligned}
& R_{I V}^{p, \alpha, \beta}(X, Y)= \\
& =\left[R^{p}\left(K_{\alpha}(X), K_{\alpha}(Y)\right)-\min ((1-\beta) w(X)+\beta w(Y), \beta w(X)+(1-\beta) w(Y)), R^{p}\left(K_{\alpha}(X), K_{\alpha}(Y)\right)\right] .
\end{aligned}
$$

Note that for $\beta=1$ (or equivalently for $\beta=0$ ), we get the class described in item (ii).

Finally, Theorem 3.6 also allows us to provide conditions to have $R_{I V}(X, Y)=1_{L}$.

Lemma 3.12. Let $R_{I V}: L([0,1])^{2} \rightarrow L([0,1])$ be defined as in Theorem 3.6. If $M(x, y)=0$ if and only if $x=y=0$, then $R_{I V}(X, Y)=1_{L}$ if and only if $X=Y$ and $w(X)=0$.

Proof. The proof is straightforward.

\subsection{Width-preserving IV aggregation functions}

In order to build width-preserving similarity measures, one possibility is to aggregate width-preserving IVREFs in a suitable way. Specifically, it is desirable that the considered aggregation function takes into account the width of the input intervals.

As a first step towards this, we recall the definition of aggregation function in the interval-valued setting.

Definition 3.13. Let $n \geq 2$. An (n-dimensional) interval-valued (IV) aggregation function in $L([0,1])$ with respect to $\leq_{L}$ is a mapping $M_{I V}:(L([0,1]))^{n} \rightarrow L([0,1])$ which verifies:

(i) $M_{I V}\left(0_{L}, \cdots, 0_{L}\right)=0_{L}$.

(ii) $M_{I V}\left(1_{L}, \cdots, 1_{L}\right)=1_{L}$.

(iii) $M_{I V}$ is a non-decreasing function with respect to $\leq_{L}$.

We say that $M_{I V}:(L([0,1]))^{n} \rightarrow L([0,1])$ is a decomposable $n$-dimensional IV aggregation function associated with $M_{L}$ and $M_{U}$, if $n$-dimensional aggregation functions $M_{L}, M_{U}:[0,1]^{n} \rightarrow[0,1]$ exist such that $M_{L} \leq M_{U}$ pointwise, and where

$$
M_{I V}\left(X_{1}, \ldots, X_{n}\right)=\left[M_{L}\left(\underline{X_{1}}, \ldots, \underline{X_{n}}\right), M_{U}\left(\overline{X_{1}}, \ldots, \overline{X_{n}}\right)\right]
$$

for all $X_{1}, \ldots, X_{n} \in L([0,1])$.

The construction of IV aggregation functions with respect to admissible orders is not a trivial task, see [1]. We devote the next results to such a construction. 
Definition $3.14([1])$. Let $c \in[0,1]$ and $\alpha \in[0,1]$. We denote by $d_{\alpha}(c)$ the maximal possible width of an interval $Z \in L([0,1])$ such that $K_{\alpha}(Z)=c$. Moreover, for any $X \in L([0,1])$, let

$$
\lambda_{\alpha}(X)=\frac{w(X)}{d_{\alpha}\left(K_{\alpha}(X)\right)}
$$

where we set $\frac{0}{0}=1$.

Proposition 3.15 ([1]). For all $\alpha \in[0,1]$ and $X \in L([0,1])$ it holds that

$$
d_{\alpha}\left(K_{\alpha}(X)\right)=\wedge\left(\frac{K_{\alpha}(X)}{\alpha}, \frac{1-K_{\alpha}(X)}{1-\alpha}\right) .
$$

where we set $\frac{r}{0}=1$ for all $r \in[0,1]$.

A construction method of IV aggregation functions w.r.t. $\leq_{\alpha, \beta}$ is proposed in the following theorem, which makes use of aggregation functions.

Theorem 3.16. Let $\alpha, \beta \in[0,1], \beta \neq \alpha$. Let $M_{1}, M_{2}:[0,1]^{n} \rightarrow[0,1]$ be aggregation functions where $M_{1}$ is strictly increasing. Then $M_{I V}:(L([0,1]))^{n} \rightarrow L([0,1])$ defined by:

$$
M_{I V}\left(X_{1}, \ldots, X_{n}\right)=Y, \quad \text { where } \quad\left\{\begin{array}{l}
K_{\alpha}(Y)=M_{1}\left(K_{\alpha}\left(X_{1}\right), \ldots, K_{\alpha}\left(X_{n}\right)\right) \\
\lambda_{\alpha}(Y)=M_{2}\left(\lambda_{\alpha}\left(X_{1}\right), \ldots, \lambda_{\alpha}\left(X_{2}\right)\right)
\end{array}\right.
$$

for all $X_{1}, \ldots, X_{n} \in L([0,1])$, is an $I V$ aggregation function with respect to $\leq_{\alpha, \beta}$.

Proof. First observe that $w(Y)=\lambda_{\alpha}(Y) d_{\alpha}\left(K_{\alpha}(Y)\right)$ and $\underline{Y}=K_{\alpha}(Y)-\alpha w(Y), \bar{Y}=K_{\alpha}(Y)+(1-\alpha) w(Y)$. Clearly, $M_{I V}$ is well defined. It suffices to show that $M_{I V}$ is an IV aggregation function.

(i) $M_{I V}\left(0_{L}, \cdots, 0_{L}\right)=Y$ where $K_{\alpha}(Y)=M_{1}(0, \ldots, 0)=0$. Moreover, if $\alpha \neq 0$, then $\lambda_{\alpha}(Y)=$ $M_{2}(1, \ldots, 1)=1$ and $w(Y)=\lambda_{\alpha}(Y) d_{\alpha}\left(K_{\alpha}(Y)\right)=1 \cdot 0=0$. If $\alpha=0$, then $\lambda_{\alpha}(Y)=M_{2}(0, \ldots, 0)=0$ and $w(Y)=\lambda_{\alpha}(Y) d_{\alpha}\left(K_{\alpha}(Y)\right)=0 \cdot 1=0$. Hence, $Y=[0,0]$.

(ii) $M_{I V}\left(1_{L}, \cdots, 1_{L}\right)=Y$ where $K_{\alpha}(Y)=M_{1}(1, \ldots, 1)=1$. Moreover, if $\alpha \neq 1$, then $\lambda_{\alpha}(Y)=$ $M_{2}(1, \ldots, 1)=1$ and $w(Y)=\lambda_{\alpha}(Y) d_{\alpha}\left(K_{\alpha}(Y)\right)=1 \cdot 0=0$. If $\alpha=1$, then $\lambda_{\alpha}(Y)=M_{2}(0, \ldots, 0)=0$ and $w(Y)=\lambda_{\alpha}(Y) d_{\alpha}\left(K_{\alpha}(Y)\right)=0 \cdot 1=0$. Hence, $Y=[1,1]$.

(iii) Let $X_{i} \leq_{\alpha, \beta} Y_{i}$ for all $i=1, \ldots, n$. Then $K_{\alpha}\left(X_{i}\right) \leq K_{\alpha}\left(Y_{i}\right)$ for all $i=1, \ldots, n$ and there are two cases:

1. There exists $j \in\{1, \ldots, n\}$ such that $K_{\alpha}\left(X_{j}\right)<K_{\alpha}\left(Y_{j}\right)$. Since $M_{1}$ is strictly increasing, it follows that

$$
M_{1}\left(K_{\alpha}\left(X_{1}\right), \ldots, K_{\alpha}\left(X_{n}\right)\right)<M_{1}\left(K_{\alpha}\left(Y_{1}\right), \ldots, K_{\alpha}\left(Y_{n}\right)\right),
$$

thus $M_{I V}\left(X_{1}, \ldots, X_{n}\right)<_{\alpha, \beta} M_{I V}\left(Y_{1}, \ldots, Y_{n}\right)$.

2. $K_{\alpha}\left(X_{i}\right)=K_{\alpha}\left(Y_{i}\right)$ for all $i=1, \ldots, n$. If $\beta>\alpha$, then $w\left(X_{i}\right) \leq w\left(Y_{i}\right)$ for all $i=1, \ldots, n$, hence $\lambda_{\alpha}\left(X_{i}\right) \leq$ $\lambda_{\alpha}\left(Y_{i}\right)$ for all $i=1, \ldots, n$, thus $M_{2}\left(\lambda_{\alpha}\left(X_{1}\right), \ldots, \lambda_{\alpha}\left(X_{n}\right)\right) \leq M_{2}\left(\lambda_{\alpha}\left(Y_{1}\right), \ldots, \lambda_{\alpha}\left(Y_{n}\right)\right)$, consequently $M_{I V}\left(X_{1}, \ldots, X_{n}\right) \leq_{\alpha, \beta} M_{I V}\left(Y_{1}, \ldots, Y_{n}\right)$. If $\beta<\alpha$, then $w\left(X_{i}\right) \geq w\left(Y_{i}\right)$ for all $i=1, \ldots, n$, hence $\lambda_{\alpha}\left(X_{i}\right) \geq \lambda_{\alpha}\left(Y_{i}\right)$ for all $i=1, \ldots, n$, thus $M_{2}\left(\lambda_{\alpha}\left(X_{1}\right), \ldots, \lambda_{\alpha}\left(X_{n}\right)\right) \geq M_{2}\left(\lambda_{\alpha}\left(Y_{1}\right), \ldots, \lambda_{\alpha}\left(Y_{n}\right)\right)$. As a consequence, $M_{I V}\left(X_{1}, \ldots, X_{n}\right) \leq_{\alpha, \beta} M_{I V}\left(Y_{1}, \ldots, Y_{n}\right)$.

The following construction methods provide IV aggregation functions w.r.t. $\leq_{\alpha, \beta}$ which preserve the width of the input intervals. First of all, given an aggregation function $M:[0,1]^{n} \rightarrow[0,1]$, the following two properties are considered:

(P1) $M\left(c x_{1}, \ldots, c x_{n}\right) \geq c M\left(x_{1}, \ldots, x_{n}\right)$ for all $c \in[0,1], x_{1}, \ldots, x_{n} \in[0,1]$. 
(P2) $M\left(x_{1}, \ldots, x_{n}\right) \leq 1-M\left(1-x_{1}, \ldots, 1-x_{n}\right)$ for all $x_{1}, \ldots, x_{n} \in[0,1]$.

Theorem 3.17. Let $\alpha, \beta \in[0,1], \beta \neq \alpha$. Let $M_{1}, M_{2}:[0,1]^{n} \rightarrow[0,1]$ be aggregation functions such that $M_{1}$ is strictly increasing, $M_{1}\left(x_{1}, \ldots, x_{n}\right) \geq M_{2}\left(x_{1}, \ldots, x_{n}\right)$ for all $x_{1}, \ldots, x_{n} \in[0,1], M_{1}$ or $M_{2}$ satisfies property (P1) and $M_{1}$ or $M_{2}$ satisfies property (P2). Then $M_{I V}:(L([0,1]))^{n} \rightarrow L([0,1])$ defined by:

$$
M_{I V}\left(X_{1}, \ldots, X_{n}\right)=Y, \quad \text { where } \quad\left\{\begin{array}{l}
K_{\alpha}(Y)=M_{1}\left(K_{\alpha}\left(X_{1}\right), \ldots, K_{\alpha}\left(X_{n}\right)\right) \\
w(Y)=M_{2}\left(w\left(X_{1}\right), \ldots, w\left(X_{n}\right)\right)
\end{array}\right.
$$

for all $X_{1}, \ldots, X_{n} \in L([0,1])$, is an IV aggregation function with respect to $\leq_{\alpha, \beta}$.

Moreover, if $M_{2}$ is idempotent, then $M_{I V}$ is w-preserving.

Proof. We first show that $M_{I V}$ is well defined. Observe that

$$
Y=[\underline{Y}, \bar{Y}]=\left[K_{\alpha}(Y)-\alpha w(Y), K_{\alpha}(Y)+(1-\alpha) w(Y)\right] .
$$

Clearly, $\underline{Y} \leq \bar{Y}$, hence we only need to prove that

1. $\underline{Y} \geq 0$ : For $\alpha=0$ we have $\underline{Y}=M_{1}\left(\underline{X_{1}}, \ldots, \underline{X_{n}}\right) \geq 0$ and for $\left.\left.\alpha \in\right] 0,1\right]$ we have

$$
K_{\alpha}(Y)=M_{1}\left(K_{\alpha}\left(X_{1}\right), \ldots, K_{\alpha}\left(X_{n}\right)\right) \geq \alpha M_{2}\left(\frac{K_{\alpha}\left(X_{1}\right)}{\alpha}, \ldots, \frac{K_{\alpha}\left(X_{n}\right)}{\alpha}\right) \geq \alpha M_{2}\left(w\left(X_{1}\right), \ldots, w\left(X_{n}\right)\right)=\alpha w(Y)
$$

where the first inequality follows from the fact that $M_{2}$ satisfies property (P1) and the second from the observation $K_{\alpha}(X)=(1-\alpha) \underline{X}+\alpha \bar{X} \geq \alpha(\bar{X}-\underline{X})=\alpha w(X)$ for all $X \in L([0,1])$.

2. $\bar{Y} \leq 1$ : For $\alpha=1$ we have $\bar{Y}=M_{1}\left(\overline{X_{1}}, \ldots, \overline{X_{n}}\right) \leq 1$ and for $\alpha \in[0,1[$ we have

$$
\begin{aligned}
& K_{\alpha}(Y)+(1-\alpha) w(Y)=M_{1}\left(K_{\alpha}\left(X_{1}\right), \ldots, K_{\alpha}\left(X_{n}\right)\right)+(1-\alpha) M_{2}\left(w\left(X_{1}\right), \ldots, w\left(X_{n}\right)\right) \leq \\
& \leq M_{1}\left(K_{\alpha}\left(X_{1}\right), \ldots, K_{\alpha}\left(X_{n}\right)\right)+(1-\alpha) M_{2}\left(\frac{1-K_{\alpha}\left(X_{1}\right)}{1-\alpha}, \ldots, \frac{1-K_{\alpha}\left(X_{n}\right)}{1-\alpha}\right) \leq \\
& \leq M_{1}\left(K_{\alpha}\left(X_{1}\right), \ldots, K_{\alpha}\left(X_{n}\right)\right)+M_{2}\left(1-K_{\alpha}\left(X_{1}\right), \ldots, 1-K_{\alpha}\left(X_{n}\right)\right) \leq \\
& \leq M_{1}\left(K_{\alpha}\left(X_{1}\right), \ldots, K_{\alpha}\left(X_{n}\right)\right)+1-M_{2}\left(K_{\alpha}\left(X_{1}\right), \ldots, K_{\alpha}\left(X_{n}\right)\right)=1
\end{aligned}
$$

where the first inequality follows from the observation $1-K_{\alpha}(X)=1-(1-\alpha) \underline{X}-\alpha \bar{X} \geq(1-\alpha)(\bar{X}-$ $\underline{X})=(1-\alpha) w(X)$ for all $X \in L([0,1])$, and the second and third ones from the assumptions of the theorem.

Now we prove that $M_{I V}$ is an IV aggregation function. (i) $M_{I V}\left(0_{L}, \cdots, 0_{L}\right)=Y$ where $K_{\alpha}(Y)=$ $M_{1}(0, \ldots, 0)=0$ and $w(Y)=M_{2}(0, \ldots, 0)=0$, hence $Y=0_{L}$. (ii) $M_{I V}\left(1_{L}, \cdots, 1_{L}\right)=Y$ where $K_{\alpha}(Y)=$ $M_{1}(1, \ldots, 1)=1$ and $w(Y)=M_{2}(0, \ldots, 0)=0$, hence $Y=1_{L}$. (iii) Let $X_{i} \leq_{\alpha, \beta} Y_{i}$ for all $i=1, \ldots, n$. Then $K_{\alpha}\left(X_{i}\right) \leq K_{\alpha}\left(Y_{i}\right)$ for all $i=1, \ldots, n$ and there are two cases:

1. There exists $j \in\{1, \ldots, n\}$ such that $K_{\alpha}\left(X_{j}\right)<K_{\alpha}\left(Y_{j}\right)$. Then

$$
M_{1}\left(K_{\alpha}\left(X_{1}\right), \ldots, K_{\alpha}\left(X_{n}\right)\right)<M_{1}\left(K_{\alpha}\left(Y_{1}\right), \ldots, K_{\alpha}\left(Y_{n}\right)\right),
$$

since $M_{1}$ is strictly increasing, thus $M_{I V}\left(X_{1}, \ldots, X_{n}\right)<_{\alpha, \beta} M_{I V}\left(Y_{1}, \ldots, Y_{n}\right)$.

2. $K_{\alpha}\left(X_{i}\right)=K_{\alpha}\left(Y_{i}\right)$ for all $i=1, \ldots, n$. If $\beta>\alpha$, then $w\left(X_{i}\right) \leq w\left(Y_{i}\right)$ for all $i=1, \ldots, n$, hence $M_{2}\left(w\left(X_{1}\right), \ldots, w\left(X_{n}\right)\right) \leq M_{2}\left(w\left(Y_{1}\right), \ldots, w\left(Y_{n}\right)\right)$, consequently $M_{I V}\left(X_{1}, \ldots, X_{n}\right) \leq_{\alpha, \beta} M_{I V}\left(Y_{1}, \ldots, Y_{n}\right)$. If $\beta<\alpha$, then $w\left(X_{i}\right) \geq w\left(Y_{i}\right)$ for all $i=1, \ldots, n$, thus $M_{2}\left(w\left(X_{1}\right), \ldots, w\left(X_{n}\right)\right) \geq M_{2}\left(w\left(Y_{1}\right), \ldots, w\left(Y_{n}\right)\right)$, consequently $M_{I V}\left(X_{1}, \ldots, X_{n}\right) \leq_{\alpha, \beta} M_{I V}\left(Y_{1}, \ldots, Y_{n}\right)$.

Finally, it is easy to check that $M_{I V}$ is $w$-preserving from the idempotency of $M_{2}$. 
To clarify our results, we consider now the case where the aggregation functions that we use to build the width-preserving IV aggregation function are given by a weighted arithmetic mean. This result will be relevant in the next section and for the application.

Proposition 3.18. Let $\left.\left.\left(v_{1}, \ldots, v_{n}\right) \in\right] 0,1\right]^{n}$ be a weighting vector with $v_{1}+\ldots+v_{n}=1$. Under the assumptions of Theorem 3.17, if $M_{1}\left(x_{1}, \ldots, x_{n}\right)=M_{2}\left(x_{1}, \ldots, x_{n}\right)=v_{1} x_{1}+\ldots+v_{n} x_{n}$ for all $x_{1}, \ldots, x_{n} \in$ $[0,1]$, then $M_{I V}$ is the decomposable IV aggregation function associated with $M_{L}$ and $M_{U}$ where $M_{L}=$ $M_{U}=M_{1}$.

Proof. Let $X_{1}, \ldots, X_{n} \in L([0,1])$ and $M_{I V}\left(X_{1}, \ldots, X_{n}\right)=Y$. According to Theorem 3.17 we have

$$
w(Y)=\sum_{i=1}^{n} v_{i} w\left(X_{i}\right) \quad \text { and } \quad K_{\alpha}(Y)=\sum_{i=1}^{n} v_{i} K_{\alpha}\left(X_{i}\right) .
$$

Taking $M_{L}=M_{U}=M_{1}$ we obtain

$$
M_{U}\left(\overline{X_{1}}, \ldots, \overline{X_{n}}\right)-M_{L}\left(\underline{X_{1}}, \ldots, \underline{X_{n}}\right)=\sum_{i=1}^{n} v_{i} \overline{X_{i}}-\sum_{i=1}^{n} v_{i} \underline{X_{i}}=\sum_{i=1}^{n} v_{i} w\left(X_{i}\right)=w(Y)
$$

and

$$
(1-\alpha) M_{L}\left(\underline{X_{1}}, \ldots, \underline{X_{n}}\right)+\alpha M_{U}\left(\overline{X_{1}}, \ldots, \overline{X_{n}}\right)=(1-\alpha) \sum_{i=1}^{n} v_{i} \underline{X_{i}}+\alpha \sum_{i=1}^{n} v_{i} \overline{X_{i}}=\sum_{i=1}^{n} v_{i} K_{\alpha}\left(X_{i}\right)=K_{\alpha}(Y) .
$$

Hence, according to Equation (7), $M_{I V}$ is decomposable and associated with $M_{L}$ and $M_{U}$.

Finally, we present some properties that hold for the functions $M_{I V}:(L([0,1]))^{n} \rightarrow L([0,1])$ defined as in Theorem 3.17 .

Lemma 3.19. Let $M_{I V}:(L([0,1]))^{n} \rightarrow L([0,1])$ be defined as in Theorem 3.17.

(i) If

- $M_{1}\left(x_{1}, \ldots, x_{n}\right)=0$ if and only if $x_{1}=\ldots=x_{n}=0$ and

- $M_{2}\left(x_{1}, \ldots, x_{n}\right)=0$ if and only if $x_{1}=\ldots=x_{n}=0$,

then $M_{I V}\left(X_{1}, \ldots, X_{n}\right)=0_{L}$ if and only if $X_{1}=\ldots=X_{n}=0_{L}$. Moreover, if $\alpha \neq 0$, then the restriction on $M_{2}$ can be skipped.

(ii) If

- $M_{1}\left(x_{1}, \ldots, x_{n}\right)=1$ if and only if $x_{1}=\ldots=x_{n}=1$ and

- $M_{2}\left(x_{1}, \ldots, x_{n}\right)=0$ if and only if $x_{1}=\ldots=x_{n}=0$,

then $M_{I V}\left(X_{1}, \ldots, X_{n}\right)=1_{L}$ if and only if $X_{1}=\ldots=X_{n}=1_{L}$. Moreover, if $\alpha \neq 1$, then the restriction on $M_{2}$ can be skipped.

(iii) $M_{I V}$ is idempotent if and only if $M_{1}$ and $M_{2}$ are idempotent.

Proof. The proof is straightforward.

Example 3.20. A function $M_{I V}:(L([0,1]))^{n} \rightarrow L([0,1])$ defined as in Theorem 3.17 , is a $w$-preserving IV aggregation function with respect to $\leq_{\alpha, \beta}$, if, for instance:

1. (i) $M_{1}\left(x_{1}, \ldots, x_{n}\right)=M_{2}\left(x_{1}, \ldots, x_{n}\right)=\frac{x_{1}+\ldots+x_{n}}{n}$ for all $x_{1}, \ldots, x_{n} \in[0,1]$, or

2. (ii) $M_{1}\left(x_{1}, \ldots, x_{n}\right)=\frac{x_{1}+\ldots+x_{n}}{n}, M_{2}\left(x_{1}, \ldots, x_{n}\right)=\min \left\{x_{1}, \ldots, x_{n}\right\}$ for all $x_{1}, \ldots, x_{n} \in[0,1]$. 


\section{Width-based interval valued similarity measures}

The same arguments that we have used to justify the introduction of IV restricted equivalence functions which take into account the width of the intervals are valid for the case of IV similarity measures. For this reason we propose the following definition.

Definition 4.1. Let $\leq_{L}$ be an order on $L([0,1])$ and $M:[0,1]^{n} \rightarrow[0,1]$ be an aggregation function. A width-based interval-valued similarity measure on $\operatorname{IVFS}(U)$ w.r.t. $\leq_{L}$, associated with $M$ is a mapping $S_{M}: \operatorname{IVF} S(U) \times \operatorname{IVFS}(U) \rightarrow L([0,1])$ such that, for all $A, B, A^{\prime}, B^{\prime} \in I V F S(U)$,

(SM1) $S_{M}(A, B)=S_{M}(B, A)$;

$(\mathrm{SM} 2) S_{M}(A, A)=\left[1-M\left(w\left(A\left(u_{1}\right)\right), \ldots, w\left(A\left(u_{n}\right)\right)\right), 1\right]$

(SM3) $S_{M}(A, B)=0_{L}$ if and only if $\left\{A\left(u_{i}\right), B\left(u_{i}\right)\right\}=\left\{0_{L}, 1_{L}\right\}$ for all $i \in\{1, \ldots, n\}$;

(SM4) If $A \subseteq A^{\prime} \subseteq B^{\prime} \subseteq B$ w.r.t. $\leq_{L}$ and $w\left(A\left(u_{i}\right)\right)=w\left(A^{\prime}\left(u_{i}\right)\right)=w\left(B^{\prime}\left(u_{i}\right)\right)=w\left(B\left(u_{i}\right)\right)$ for all $i \in$ $\{1, \ldots, n\}$, then $S_{M}(A, B) \leq_{L} S_{M}\left(A^{\prime}, B^{\prime}\right)$, where, for $A, B \in I V F S(U), A \subseteq B$ w.r.t. $\leq_{L}$ if $A\left(u_{i}\right) \leq_{L}$ $B\left(u_{i}\right)$ for every $u_{i} \in U$.

The definition is motivated by that given in [11]. However, the second axiom is changed in line with Definition 3.1 and the fourth axiom is relaxed in a similar way as the fourth axiom in Definition 3.1.

Now, a construction method of IV similarity measure by aggregation of IVREFs is given.

Recall that an aggregation function $M:[0,1]^{n} \rightarrow[0,1]$ is called self-dual with respect to the standard negation if

$$
M\left(x_{1}, \ldots, x_{n}\right)=1-M\left(1-x_{1}, \ldots, 1-x_{n}\right)
$$

for all $x_{1}, \ldots, x_{n} \in[0,1]$.

Theorem 4.2. Let $M_{I V}:(L([0,1]))^{n} \rightarrow L([0,1])$ be a decomposable $I V$ aggregation function w.r.t. $\leq_{L}$ associated with $M_{L}$ and $M_{U}$ where $M_{L}$ is self-dual, and let $M_{I V}\left(X_{1}, \ldots, X_{n}\right)=0_{L}$ if and only if $X_{1}=$ $\ldots=X_{n}=0_{L}$. Let $R_{I V}: L([0,1])^{2} \rightarrow L([0,1])$ be an $I V$ restricted equivalence function w.r.t. $\leq_{L}$. Then the function $S_{M_{L}}: \operatorname{IVFS}(U) \times \operatorname{IVFS}(U) \rightarrow L([0,1])$ defined by:

$$
S_{M_{L}}(A, B)=M_{I V}\left(R_{I V}\left(A\left(u_{1}\right), B\left(u_{1}\right)\right), \ldots, R_{I V}\left(A\left(u_{n}\right), B\left(u_{n}\right)\right)\right)
$$

for all $A, B \in I V F S(U)$ is a width-based IV similarity measure on IVFS $(U)$ w.r.t. $\leq_{L}$ associated with $M_{L}$.

Proof. (SM1) Directly follows from Axiom 3 of Definition 3.1.

(SM2) Observe that

$$
\begin{gathered}
S_{M_{L}}(A, A)=M_{I V}\left(R_{I V}\left(A\left(u_{1}\right), A\left(u_{1}\right)\right), \ldots, R_{I V}\left(A\left(u_{n}\right), A\left(u_{n}\right)\right)\right)= \\
=M_{I V}\left(\left[1-w\left(A\left(u_{1}\right)\right), 1\right], \ldots,\left[1-w\left(A\left(u_{n}\right)\right), 1\right]\right)=\left[M_{L}\left(1-w\left(A\left(u_{1}\right)\right), \ldots, 1-w\left(A\left(u_{n}\right)\right)\right), 1\right]= \\
=\left[1-M_{L}\left(w\left(A\left(u_{1}\right)\right), \ldots, w\left(A\left(u_{n}\right)\right)\right), 1\right],
\end{gathered}
$$

where the second equality follows from Axiom 2 of Definition 3.1, the third from the fact that $M_{I V}$ is decomposable associated with $M_{L}, M_{U}$ and the last from the self-duality of $M_{L}$.

(SM3) Since $S_{M_{L}}(A, B)=0_{L}$ if and only if $R_{I V}\left(A\left(u_{1}\right), B\left(u_{1}\right)\right)=0_{L}$ for all $i \in\{1, \ldots, n\}$, which only holds if $\left\{A\left(u_{i}\right), B\left(u_{i}\right)\right\}=\left\{0_{L}, 1_{L}\right\}$ for all $i \in\{1, \ldots, n\}$, (SM3) is satisfied.

(SM4) Follows from Axiom 4 of Definition 3.1 and the monotonicity of $M_{I V}$. 
Example 4.3. Let us consider the IVREF $R_{\text {epis }}$ given in Example $3.3(\mathrm{i}), M_{L}\left(x_{1}, \ldots, x_{n}\right)=\frac{1}{n} \sum_{i=1}^{n} x_{i}$ and $M_{U}\left(x_{1}, \ldots, x_{n}\right)=\max \left(x_{1}, \ldots, x_{n}\right)$. Then the assumptions of Theorem 4.2 are satisfied and we obtain the following width-based IV similarity measure on $\operatorname{IVFS}(U)$ :

$$
\begin{aligned}
S(A, B)= & {\left[\frac{1}{n} \sum_{i=1}^{n}\left(1-\max \left(\overline{A\left(u_{i}\right)}-\underline{B\left(u_{i}\right)}, \overline{B\left(u_{i}\right)}-\underline{A\left(u_{i}\right)}\right)\right)\right.} \\
& \left., \max \left(1-\max \left(0, \max \left(\underline{A\left(u_{i}\right)}, \underline{B\left(u_{i}\right)}\right)-\min \left(\overline{A\left(u_{i}\right)}, \overline{B\left(u_{i}\right)}\right)\right)\right)\right] .
\end{aligned}
$$

We now study the conditions under which the IVREF $R_{I V}$ given by Theorem 3.6 and the IV aggregation function $M_{I V}$ given by Theorem 3.17 can be applied in the previous theorem to obtain an IV similarity measure that preserves the width of intervals.

Corollary 4.4. Let $\alpha, \beta \in] 0,1\left[\right.$ where $\beta \neq \alpha$. Let $\left.\left.\left(v_{1}, \ldots, v_{n}\right) \in\right] 0,1\right]^{n}$ be a weighting vector such that $v_{1}+\ldots+v_{n}=1$ and let $M_{I V}:(L([0,1]))^{n} \rightarrow L([0,1])$ be the IV aggregation function w.r.t. $\leq_{\alpha, \beta}$ defined as in Theorem 3.17 where $M_{1}\left(x_{1}, \ldots, x_{n}\right)=M_{2}\left(x_{1}, \ldots, x_{n}\right)=v_{1} x_{1}+\ldots+v_{n} x_{n}$ for all $x_{1}, \ldots, x_{n} \in$ $[0,1]$. Let $R_{I V}:(L([0,1]))^{2} \rightarrow L([0,1])$ be an IVREF defined as in Theorem 3.6. Then the function $S_{M}: \operatorname{IVFS}(U) \times \operatorname{IVFS}(U) \rightarrow L([0,1])$ defined by:

$$
S_{M}(A, B)=M_{I V}\left(R_{I V}\left(A\left(u_{1}\right), B\left(u_{1}\right)\right), \ldots, R_{I V}\left(A\left(u_{n}\right), B\left(u_{n}\right)\right)\right),
$$

for all $A, B \in I V F S(U)$, is a width-based IV similarity measure on $I V F S(U)$ w.r.t. $\leq_{L}$ associated with $M_{1}$. Moreover, $S_{M}$ satisfies the following for all $A, B \in I V F S(U)$ :

$$
w\left(S_{M}(A, B)\right)=w\left(A\left(u_{1}\right)\right) \quad \text { whenever } \quad w\left(A\left(u_{1}\right)\right)=w\left(B\left(u_{1}\right)\right)=\ldots=w\left(A\left(u_{n}\right)\right)=w\left(B\left(u_{n}\right)\right) .
$$

Proof. Observe that, by Prop. 3.18, $M_{I V}$ is the decomposable IV aggregation function associated with $M_{L}$, $M_{U}$ where $M_{L}=M_{U}=M_{1}$. Moreover, by Lemma 3.19 we have that $M_{I V}\left(X_{1}, \ldots, X_{n}\right)=0_{L}$ if and only if $X_{1}=\ldots=X_{n}=0_{L}$. Since a weighted arithmetic mean is self-dual and idempotent, from Theorem 4.2 it follows that $S_{M}$ is a width-based IV similarity measure associated with $M_{1}$. Finally, since $M_{I V}$ and $R_{I V}$ are $w$-preserving, we have (8).

Example 4.5. Let $\left.\left.\left(v_{1}, \ldots, v_{n}\right) \in\right] 0,1\right]^{n}$ be a weighting vector with $v_{1}+\ldots+v_{n}=1$. Consider the IVREF given by Example 3.11 (iii) for $p=1, \alpha=0.5, \beta=1$; and the IV aggregation function defined as in Theorem 3.17 for $\alpha=0.5$ (and $\beta=1$, for instance) and $M_{1}\left(x_{1}, \ldots, x_{n}\right)=M_{2}\left(x_{1}, \ldots, x_{n}\right)=v_{1} x_{1}+\ldots+v_{n} x_{n}$. Then, applying Corollary 4.4, we obtain a width-based IV similarity measure $S_{M_{1}}$ on $I V F S(U)$ w.r.t. $\leq_{L}$ associated with $M_{1}$; moreover, $S_{M_{1}}$ satisfies (8).

As a special case, which is later used in the stereo matching application in the following section, we give an expression of $S_{M_{1}}$ for weighting vector $\left(\frac{1}{n}, \ldots, \frac{1}{n}\right)$. Note that, since the considered IV aggregation function is decomposable, the expression can be simplified significantly (see Lemma 3.18):

$$
\begin{aligned}
S_{M_{1}}(A, B)= & {\left[1-\frac{\sum_{i=1}^{n}\left|\frac{A\left(u_{i}\right)}{1} \overline{A\left(u_{i}\right)}-\underline{B\left(u_{i}\right)}-\overline{B\left(u_{i}\right)}\right|}{2 n}-\frac{\sum_{i=1}^{n} \min \left(w\left(A\left(u_{i}\right)\right), w\left(B\left(u_{i}\right)\right)\right)}{n},\right.} \\
& \left., 1-\frac{\sum_{i=1}^{n}\left|\frac{A\left(u_{i}\right)}{1}+\overline{A\left(u_{i}\right)}-\underline{B\left(u_{i}\right)}-\overline{B\left(u_{i}\right)}\right|}{2 n}\right] .
\end{aligned}
$$

Theorem 4.6. Let $M_{I V}:(L([0,1]))^{n} \rightarrow L([0,1])$ be an $I V$ aggregation function w.r.t. $\leq_{L}$ satisfying $M_{I V}\left(X_{1}, \ldots, X_{n}\right)=1_{L}$ if and only if $X_{1}=\ldots=X_{n}=1_{L}$ and $M_{I V}\left(X_{1}, \ldots, X_{n}\right)=0_{L}$ if and only if 


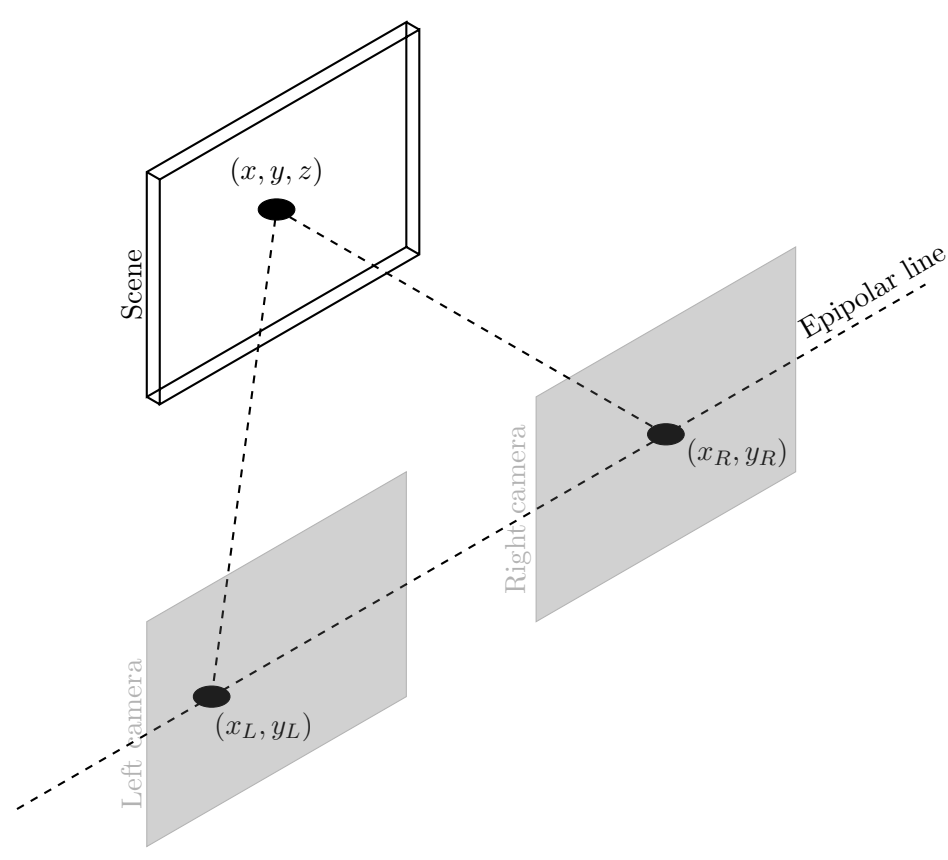

Figure 1: Schema of epipolar geometry for stereo vision.

$X_{1}=\ldots=X_{n}=0_{L}$. Let $R_{I V}:(L([0,1]))^{2} \rightarrow L([0,1])$ be a function satisfying axioms 1, 3, 4 from Definition 3.1 and $R_{I V}(X, Y)=1_{L}$ if and only if $X=Y$ and $w(X)=0$ for all $X, Y \in L([0,1])$. Then the function $S_{M}: \operatorname{IVFS}(U) \times \operatorname{IVFS}(U) \rightarrow L([0,1])$ defined by:

$$
S_{M}(A, B)=M_{I V}\left(R_{I V}\left(A\left(u_{1}\right), B\left(u_{1}\right)\right), \ldots, R_{I V}\left(A\left(u_{n}\right), B\left(u_{n}\right)\right)\right)
$$

for all $A, B \in I V F S(U)$ satisfies axioms (SM1), (SM3), (SM4) and

$\left(S M 2^{\prime}\right) S(A, B)=1_{L}$ if and only if $A=B$ and $A, B \in F S(U)$.

Proof. The proof is obtained just by a straight calculation.

Remark 4.7. Note that replacing axiom (SM2) in Definition 4.1 by Axiom (SM2'), we get an alternative approach to IV similarity measures which takes the width of intervals into account and is in the line with the ideas stated after Definition 3.1.

In the next Section we apply our developments to a stereo matching problem.

\section{Stereo matching with $w$-preserving interval-valued restricted equivalence functions}

Stereo vision arises as a model to capture the information around us in the same way as human vision does. Human visual perception is based on the formation of 3D images of the environment. Each eye captures a different scene, and from both, our brain builds a 3D image of the world around us. In stereo vision, human vision is mimicked by means of two cameras pointing at the same scene, such that each camera acts effectively as one 'eye'. In order to obtain a perception of depth from these two images, the so-called correspondence between stereo images is used. Correspondence between stereo images is one of the main problems in computer vision $[37,45]$ and it is very relevant in applications such as 3D scene reconstruction, autonomous movement or robotics.

The camera system used to catch stereo images is arranged according to an epipolar geometry, such that each point of the cameras lays on a plane, as shown in Fig. 1. Points captured from the scene by each of 
the cameras corresponding to the same point in the space are called corresponding points. Finding these corresponding points in the captured images is not a trivial task. Corresponding points may be affected by noise, occlusions or distortion during the capturing procedure. This task becomes easier if it is required that points in the captured images are subject to the epipolar restriction, i.e., each point should be in its corresponding epipolar line. This requirement implies that the search for the best correspondence should only be done along one of the dimensions of the image (horizontal axis).

Once the corresponding points have been located, a disparity map can be calculated. This map represents the depth of the objects in the image. Each disparity value is obtained by calculating the difference between the positions of the corresponding points along the horizontal axis. In the next subsection, we discuss a specific way for calculating the disparity map. Finally, we will apply the proposed IV similarity measures, taking into the width of the intervals into account.

\subsection{Methods for calculating the disparity map}

For us, an image is a function $f: X \times Y=\{1, \ldots, r\} \times\{1, \ldots, c\} \mapsto L$, where $r$ represents the number of rows in the image, that is, its height; $c$ is the number of columns in the image, that is, its width; and $L$ is a finite lattice whose elements are used to capture the intensities of the pixels in the image. Different choices of $L$ allow the representation of different types of images. In particular, if we take $L=\{0,1\}$, we represent black and white images, whereas for greyscale images we take $L=\{0, \ldots, 255\}$, and for color images in the RGB color space we take $L=\{0, \ldots, 255\}^{3}$.

In order to do the correspondence procedure and to build the disparity map, we use the block-correspondence method. This technique consists of selecting a window of size $n \times m$ in the right image and calculating the similarity of this window with each of the windows of the same size, centered at pixels in the same epipolar line in the left image. To calculate this similarity, classical measures have been usually considered. In stereo vision, the most common ones are SSD [40], SAD [44], NCC [22] o ZNCC [38]. Furthermore, some authors have used fuzzy measures [39]. Among the latter, we find some studies which consider extensions of fuzzy sets, in particular interval-valued fuzzy sets [24] or Atanassov intuitionistic fuzzy sets [33].

In Algorithm 1 we present a method for constructing a disparity map in an RGB image a using IVsimilarity measures.

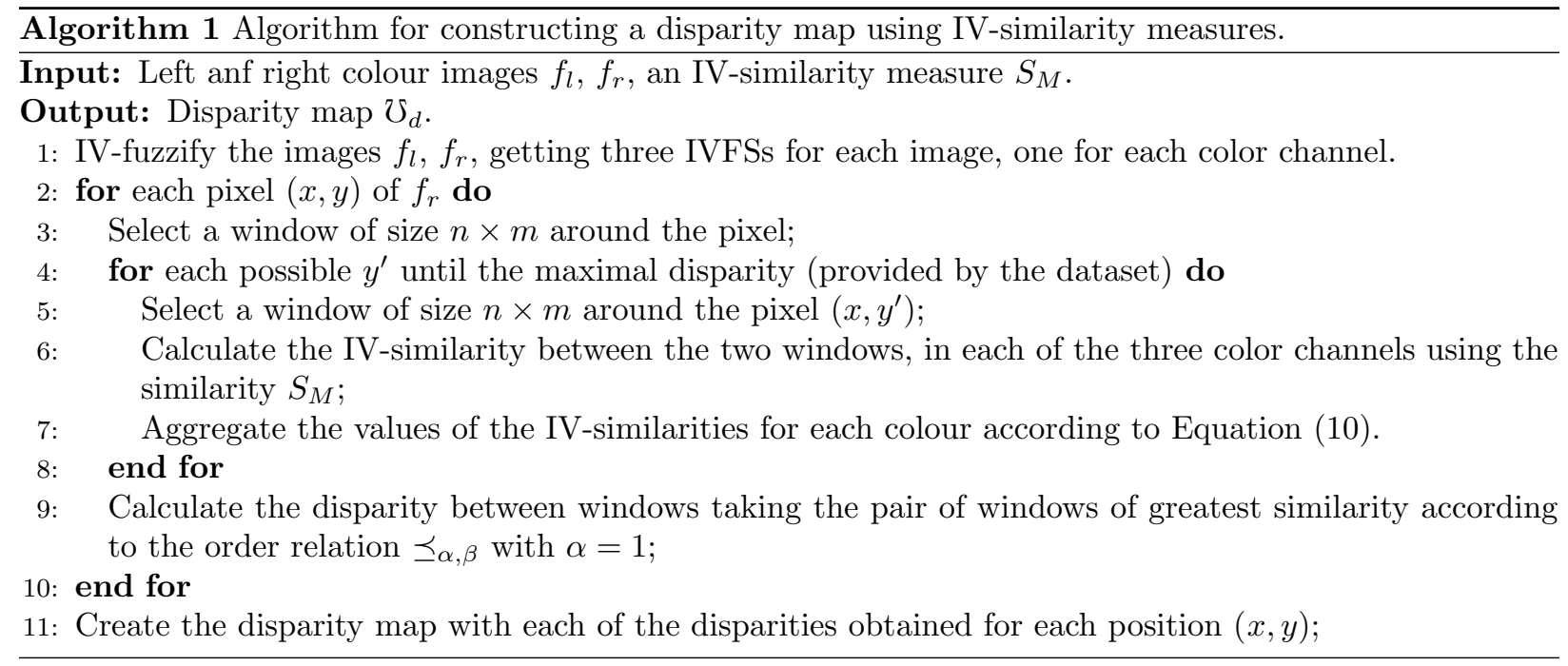

For steps 3 and 5 we proceed as indicated in Fig. 2, comparing the window in the right image to the different windows in the left image. To calculate the similarity between intervals, in step 6 we use the expression of width-based IV similarity proposed in Eq (9). Besides, as we take into account the color information in the images, in step 7 we aggregate the information as suggested in [20], by means of the 

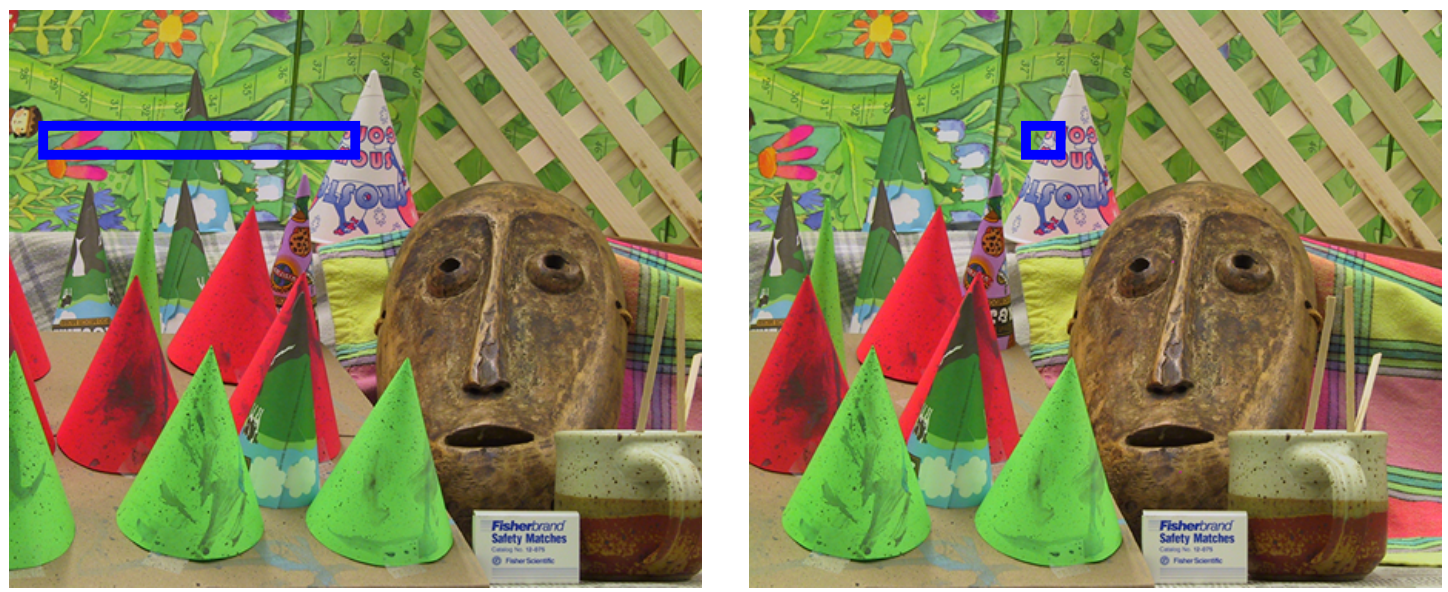

Figure 2: Choice of windows for calculating the IV similarity and the disparity .

expression:

$$
S_{M T}(A, B)=0.299 \cdot S_{M_{R}}(A, B)+0.587 \cdot S_{M_{G}}(A, B)+0.114 \cdot S_{M_{B}}(A, B)
$$

where $S_{M_{R}}(A, B), S_{M_{G}}(A, B), S_{M_{B}}(A, B)$ represent the similarity values for each of the color channels calculated using a width-based IV similarity measure $S_{M}$. Beyond the weighted mean (wMean) considered in Eq. (10), in the experiments, a number of other aggregation functions are also used to merge the similarity values, including the arithmetic mean (mean), the product (prod), the geometric mean (gmean), the harmonic mean (hmean), the median (median), the maximum (max) and the minimum (min). Finally, as in [24], for all the images we consider windows of size $7 \times 11$.

\subsection{IV-fuzzification}

A digital image is the result of a discretization of a real world scene. In order to represent such digital images, fuzzy sets have been used in the literature. However, due to the uncertainty which is inherent to the process of discretization, it is very hard to provide an accurate (real-valued) membership value for each pixel. In this sense, the use of interval-valued fuzzy sets allow us to consider such uncertainty by means of the width of the membership intervals associated to each pixel, as the precise (real-valued) fuzzy membership value is an unknown value considered to be inside the provided membership interval. For these reasons, and as it was done in [24], we represent the images by means of interval-valued fuzzy sets, assigning an interval-valued membership to each pixel. In particular, we are going to build the IVFSs representing a given image from different membership functions, as was done in [24]. It is worth mentioning that this IV-fuzzification method has been successfully used in image segmentation problems, obtaining results better than those methods which only take into account one single membership function.

Given $k$ restricted equivalence functions $R_{i}$, the procedure to get an IV-fuzzified image is the following:

- Consider an image $f$ with $L$ intensity values.

- For each level of intensity $t,(t=0, t=1, \ldots, L-1)$ :

* Build $k$ fuzzy sets $Q_{t}^{1} \ldots Q_{t}^{k}$ where for each $i=1 \ldots k$

$$
\begin{array}{r}
Q_{t}^{i}=\left\{\left(q, \mu_{Q_{t}^{i}}(q) \mid q \in\{0,1, \ldots L-1\}\right)\right\}, \text { with } \\
\mu_{Q_{t}^{i}}(q)= \begin{cases}R_{i}\left(\frac{q}{L-1}, \frac{m_{b}(t)}{L-1}\right), & \text { si } q \leq t . \\
R_{i}\left(\frac{q}{L-1}, \frac{m_{o}(t)}{L-1}\right), & \text { si } q>t .\end{cases}
\end{array}
$$


where $m_{o}(t)$ and $m_{b}(t)$ are the mean intensities of the object and the background, given by

$$
\begin{aligned}
& m_{o}(t)=\frac{\sum_{q=0}^{t} q \cdot h(q)}{\sum_{q=0}^{t=0} h(q)} \\
& m_{b}(t)=\frac{\sum_{q=t+1}^{L-1} q \cdot h(q)}{\sum_{q=t+1}^{L-1} h(q)}
\end{aligned}
$$

where $h(q)$ denotes the number of pixels with intensity $q$.

* For each level of intensity $t$, build an IVFS $\tilde{Q}_{t}$ from the fuzzy sets $Q_{t}^{1} \ldots Q_{t}^{k}$, taking, for each $q \in\{0, \ldots, L-1\}$,

$\mu_{\tilde{Q}_{t}}(q)=\left[T\left(\mu_{Q_{t}^{1}}(q), \ldots, \mu_{Q_{t}^{k}}(q)\right), S\left(\mu_{Q_{t}^{1}}(q), \ldots, \mu_{Q_{t}^{k}}(q)\right)\right]$ with $T$ a t-norm and $S$ a tconorm.

* Calculate the entropy $\epsilon_{F}$ of each of the $L$ interval valued fuzzy sets $\tilde{Q}_{t}$ using the expression: $\epsilon_{F}(\tilde{A})=\frac{1}{N} \sum_{i=1}^{N}\left(\bar{\mu}_{\tilde{A}}\left(x_{i}\right)-\underline{\mu}_{\tilde{A}}\left(x_{i}\right)\right)$, proposed in [35]

For representing the image $f$, select the IVFS $\tilde{Q}_{t}$ of smallest entropy.

Note that we take as best interval valued fuzzy set for representing the image the one of smallest entropy $\epsilon_{F}$. This is so because this interval-valued fuzzy set displays the smallest amount of uncertainty in order to build the precise value of membership for the pixels.

In the experimentation, we use the configuration proposed in the first experiment in [24], i.e., we take $k=2$ and the restricted equivalence functions:

$$
\left\{\begin{array}{l}
R_{1}(x, y)=1-|x-y| \\
R_{2}(x, y)=1-|\sqrt[10]{x}-\sqrt[10]{y}|
\end{array}\right.
$$

\section{3. w-preserving IVREF Stereo Matching}

To test the behaviour of the proposed width-depending IV similarity measure, in this work we use the Middlebury dataset [37], composed of the images in Fig. 3. One of the advantages of this dataset is that each pair of images is associated with a model of the disparity map (groundtruth) provided by an expert.

The evaluation of the error in a stereo matching procedure is done by calculating the absolute error percentage between the obtained disparity maps and the groundtruth provided by the dataset. To measure the error percentage, three types of regions, defined by the dataset, are considered:

- nonocc: considers only those pixels that are not occluded (occluded pixels are those which only appear in one of the images)

- all: all the pixels of the image are considered.

- disc: pixels near the discontinuous regions are considered.

In Fig. 4, an example of the evaluation regions is shown. For each one of the regions only pixels labelled in white are considered as part of the error computation.

\subsection{Comparison of the proposed interval methods}

We compare Algorithm 1 using our width-based IV similarity measure with the one proposed in [24], which also makes use of interval-valued fuzzy sets but without taking into account the width of the intervals.

Table 1 shows the results obtained in the experiments, highlighting the best result in bold. In this table, IVREF denotes the method using Eq. (9) and the proposed definitions of $w$-preserving IV restricted equivalence function and similarity, and IVFS denotes the method in [24]. Furthermore, the aggregation function applied to merge the colour channels is specified after the terms IVREF or IVFS, respectively. In addition, we also consider grayscale images.

We can observe that the new similarity method outperforms - in respect to the total error, the one based on fuzzy sets presented in [24], even with different aggregation functions for colour merging. The only 

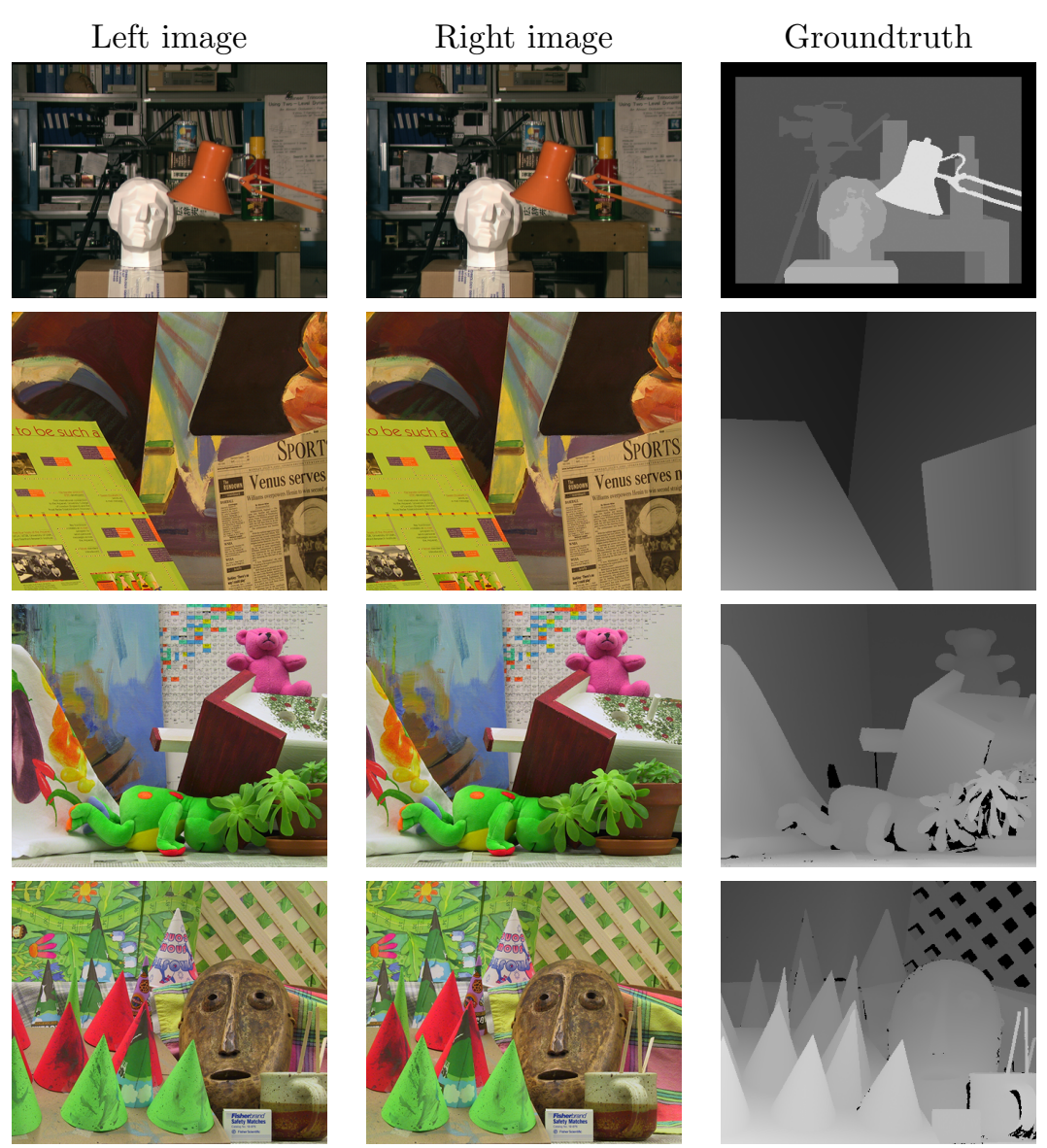

Figure 3: Left and right images and groundtruth image proposed in the dataset Middlebury.

Nonocc

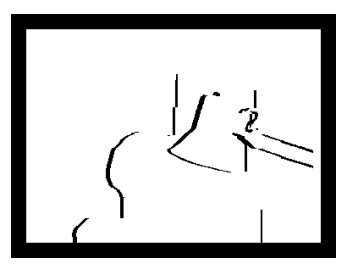

All
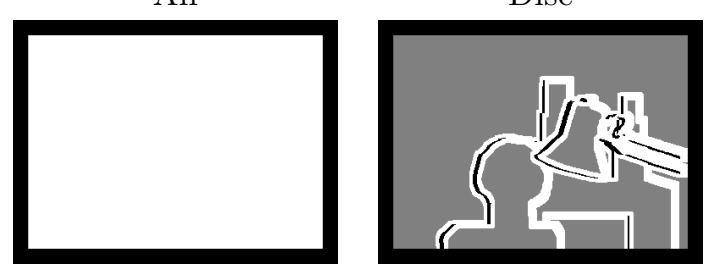

Figure 4: Regions considered for the evaluation of a disparity map 


\begin{tabular}{|c|c|c|c|c|c|c|c|c|c|c|c|c|c|}
\hline \multirow{2}{*}{ Algorithm } & \multicolumn{3}{|c|}{ Tsukuba } & \multicolumn{3}{|c|}{ Venus } & \multicolumn{3}{|c|}{ Teddy } & \multicolumn{3}{|c|}{ Cones } & \multirow{2}{*}{$\% \mathrm{Et}$} \\
\hline & \%noocc & \%all & \%disc & \%noocc & $\%$ all & \%disc & \%noocc & \%all & \%disc & \%noocc & $\%$ all & \%disc & \\
\hline IVREF wMean & 5.70 & 7.56 & 19.20 & 9.00 & 10.51 & 35.61 & 14.90 & 23.61 & 35.02 & 7.46 & 17.44 & 20.69 & 17.23 \\
\hline IVFS wMean & 8.02 & 9.94 & 17.12 & 14.98 & 16.38 & 32.12 & 16.60 & 25.16 & 33.44 & 7.79 & 17.97 & 19.34 & 18.24 \\
\hline IVREF grayscale & 6.29 & 8.22 & 20.46 & 7.48 & 9.01 & 34.87 & 15.85 & 24.43 & 33.67 & 8.16 & 18.02 & 22.16 & 17.38 \\
\hline IVFS grayscale & 8.87 & 10.81 & 18.84 & 11.71 & 13.19 & 32.13 & 18.91 & 27.21 & 33.24 & 9.54 & 19.54 & 22.76 & 18.90 \\
\hline IVREF mean & 6.06 & 7.86 & 19.37 & 11.16 & 12.62 & 35.53 & 15.75 & 24.37 & 35.62 & 7.80 & 17.75 & 21.34 & 17.94 \\
\hline IVFS mean & 8.44 & 10.30 & 17.29 & 17.92 & 19.28 & 32.73 & 18.61 & 26.96 & 34.66 & 8.34 & 18.48 & 20.02 & 19.42 \\
\hline IVREF prod & 6.07 & 7.86 & 19.37 & 11.18 & 12.64 & 35.54 & 15.79 & 24.41 & 35.67 & 7.80 & 17.75 & 21.33 & 17.95 \\
\hline IVFS prod & 8.44 & 10.31 & 17.31 & 17.94 & 19.30 & 32.74 & 18.69 & 27.03 & 34.76 & 8.34 & 18.49 & 20.02 & 19.45 \\
\hline IVREF gmean & 6.07 & 7.86 & 19.37 & 11.18 & 12.64 & 35.54 & 15.79 & 24.41 & 35.67 & 7.80 & 17.75 & 21.33 & 17.95 \\
\hline IVFS gmean & 8.44 & 10.31 & 17.31 & 17.94 & 19.30 & 32.74 & 18.69 & 27.03 & 34.76 & 8.34 & 18.49 & 20.02 & 19.45 \\
\hline IVREF hmean & 6.07 & 7.86 & 19.39 & 11.20 & 12.66 & 35.51 & 15.84 & 24.46 & 35.73 & 7.81 & 17.75 & 21.35 & 17.97 \\
\hline IVFS hmean & 8.45 & 10.31 & 17.32 & 17.96 & 19.32 & 32.74 & 18.76 & 27.09 & 34.82 & 8.35 & 18.50 & 20.04 & 19.47 \\
\hline IVREF max & 6.25 & 7.98 & 20.42 & 10.61 & 12.10 & 35.28 & 16.42 & 24.92 & 35.79 & 9.72 & 19.53 & 24.03 & 18.59 \\
\hline IVFS max & 9.13 & 10.90 & 19.06 & 18.68 & 20.05 & 34.57 & 21.29 & 29.35 & 35.63 & 11.83 & 21.63 & 25.50 & 21.47 \\
\hline IVREF median & 7.07 & 8.89 & 21.03 & 14.09 & 15.50 & 35.99 & 17.49 & 25.98 & 35.98 & 9.24 & 19.07 & 23.89 & 19.52 \\
\hline IVFS median & 9.72 & 11.60 & 18.93 & 20.87 & 22.20 & 33.81 & 20.98 & 29.11 & 34.53 & 11.04 & 20.91 & 23.71 & 21.45 \\
\hline IVREF min & 7.63 & 9.45 & 20.30 & 22.49 & 23.76 & 37.06 & 29.21 & 36.37 & 43.77 & 12.51 & 21.96 & 26.79 & 24.27 \\
\hline IVFS min & 11.05 & 12.91 & 18.63 & 30.60 & 31.74 & 36.52 & 35.41 & 41.97 & 45.50 & 17.01 & 26.19 & 26.83 & 27.86 \\
\hline
\end{tabular}

Table 1: Comparison of the two methods, IVFS and IVREF, with different aggregation functions to merge colour information. The first column represents the technique and aggregation used. The remaining columns represent for each image the percentage of incorrect disparities obtained for each evaluated region. Finally, the last column represents the mean error.

exceptions to this are when the median or min are used. It is worth mentioning that although the best performance is achieved when the weighted mean is used in respect to the overall result, in the case of the Venus, the image the performance obtained with grayscale images is considerably better for non occluded regions and for all regions.

\subsection{Comparison of the proposed method with other methods which do not use intervals}

When we compare the best results (using the weighted mean) with the classical methods in the literature, as SSD, SAD, NCC, ZNCC and fuzzy measures (FUZZY), we clearly see in Table 2 that the new proposal gets globally better results.

The proposed method obtains the best results in the images Tsukuba and Cones, whereas for the other two ones, (Venus and Teddy) it remains below the ZNCC method using grayscale images. As we can see, our method outperforms the other ones in non occluded regions, in images where there is a wide variety of colour tones (Tsukuba and Cones). In regions near discontinuities, the best approach is the FUZZY similarity approach, with grayscale images. In Fig. 5, we show the approaches that obtain the best results in each of the images and regions. Note also that our method leads to disparity maps with less incorrect disparities, mainly in the case of Tsukuba and Cones. Nevertheless, the disparity values obtained tend to fail at getting the right value near discontinuous pixels. In these regions of discontinuity, better disparity 


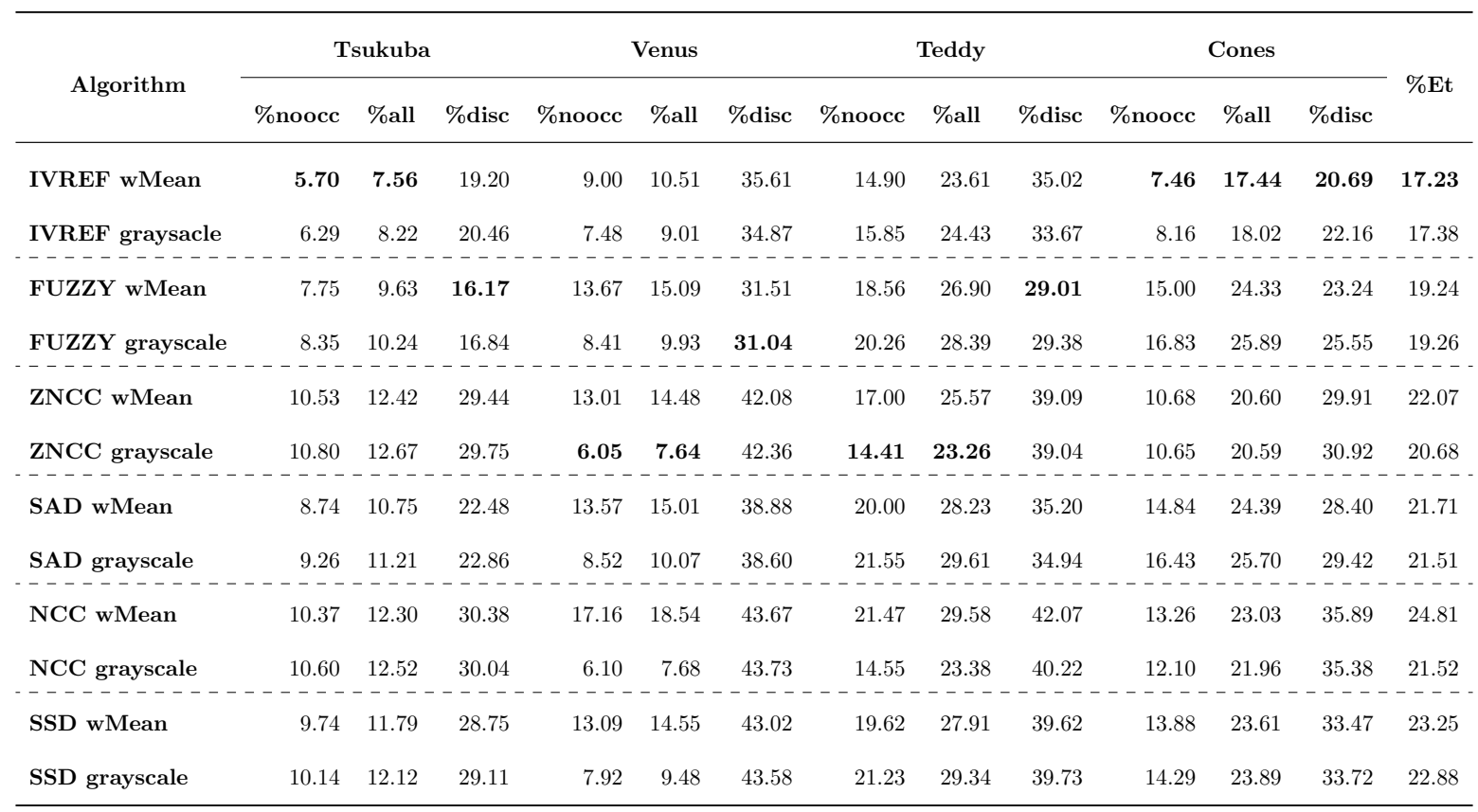

Table 2: Comparison of our similarity measure using the weighted mean for colour aggregation with the classical approaches SSD, SAD, ZNCC and Fuzzy similarity. The first column represents the technique and aggregation operator used. The remaining columns represent for each image the percentage of incorrect disparities obtained for each evaluated region. Finally, the last column represents the mean error.

values are obtained with the FUZZY approach, except in the case of Cones, where these regions get worse values.

Note that the inclusion of colour information in the process is useful in some cases where the correct value is not clear - here, it offsets some ambiguities. Despite being the best performer, our method still exhibits incorrect disparity values. This is because we have applied a basic methodology without refinement. The results shown are those from the raw disparity maps.

\subsection{Illustrative example with refinement steps}

As an illustrative example and to show the possibility of improvement of the proposed method, we discuss here an example using some refinement steps.

For the sake of this experiment we have chosen steps 3 and 5 of Algorithm 1 , a window of size $3 \times 3$, coupled with an Exponential step aggregation function [43] and a Cost Guided filter [26]. Finally we use an LRC [37] to check the raw disparity map for possible outliers.

We can observe that most of the outliers are removed, obtaining a more homogeneous disparity map, although, some objects in the images lose their shape. For example, in the case of the image Tsukuba in Fig. 6 the electrical wire in the lamp disapears. Also, the use of a filter in the smoothing process makes the shapes of the objects better defined just prior to obtaining the disparity value. In the case of the Tsukuba and Cones images, the lamp and the cones have almost recovered their shape.

The quantitative results exposed in Table 3 confirm the benefits of using refinement processes during the similarity computation and in the raw disparity map processing. We see that the global error goes down to $10.67 \%$ and that in all the analysed images and in all the regions used for error measuring, the refinement improves the results. The most significant decrease occurs in the Venus image, where the disparity values homogeneize the image and outliers almost disapear. 

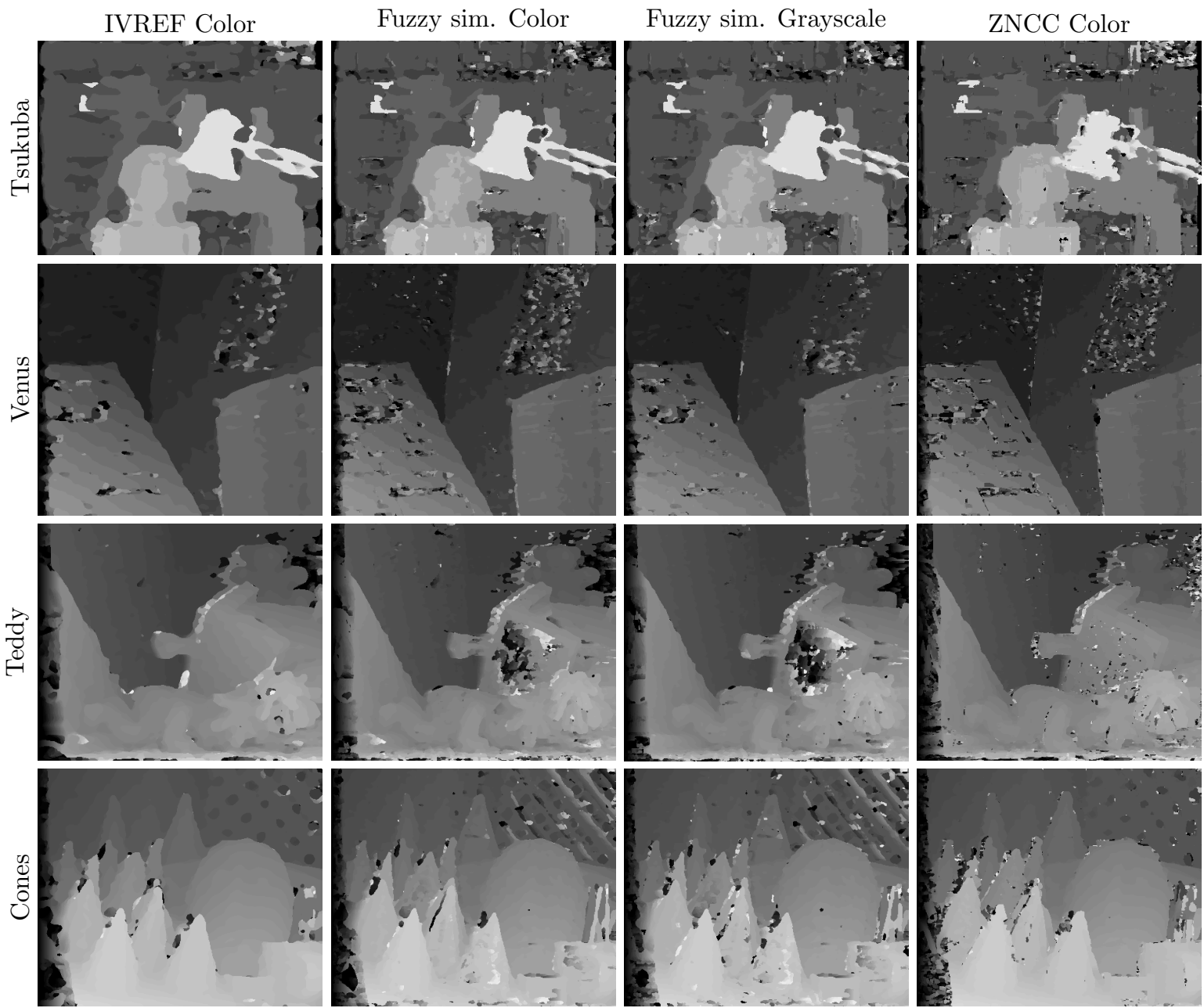

Figure 5: Disparity maps with the best obtained results. That is, IVREF aggregating colour with the weighted mean, FUZZY with color aggregation and grayscale and ZNCC with grayscale images.

\begin{tabular}{|c|c|c|c|c|c|c|c|c|c|c|c|c|c|}
\hline \multirow{2}{*}{ Algorithm } & \multicolumn{3}{|c|}{ Tsukuba } & \multicolumn{3}{|c|}{ Venus } & \multicolumn{3}{|c|}{ Teddy } & \multicolumn{3}{|c|}{ Cones } & \multirow{2}{*}{$\% \mathrm{Et}$} \\
\hline & \%noocc & \%all & \%disc & \%noocc & \%all & \%disc & \%noocc & \%all & \%disc & \%noocc & \%all & \%disc & \\
\hline IVREF wMean & 5.70 & 7.56 & 19.20 & 9.00 & 10.51 & 35.61 & 14.90 & 23.61 & 35.02 & 7.46 & 17.44 & 20.69 & 17.23 \\
\hline IVREF modified & 3.10 & 3.76 & 13.42 & 2.43 & 2.99 & 19.79 & 11.49 & 17.14 & 26.09 & 4.72 & 10.74 & 12.34 & 10.67 \\
\hline
\end{tabular}

Table 3: Comparison of our similarity measure without post-processing and our same method combined with exponential cost aggregation and cost guided filtering in addition to LRC check. 

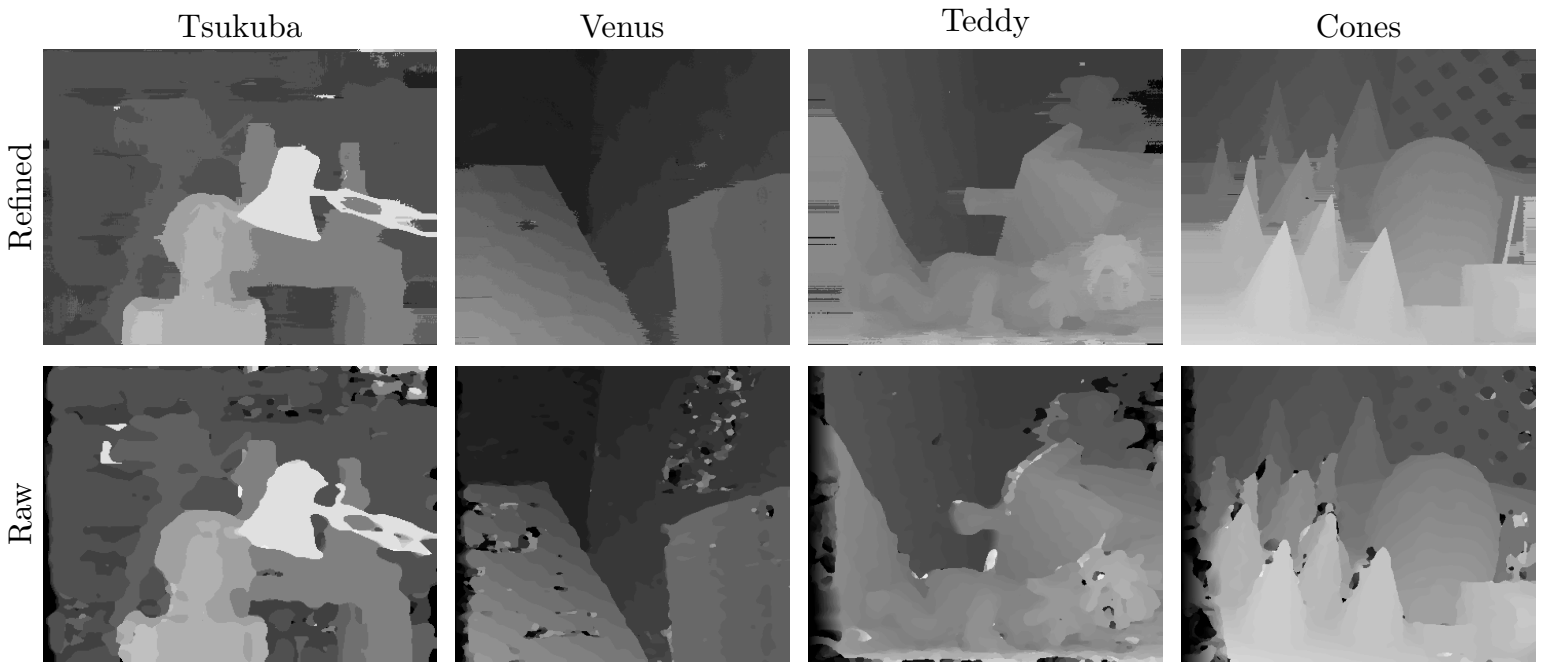

Figure 6: Comparison of the raw disparity maps obtained with raw IVREF and after applying refinement and outlier detection techniques

\section{Conclusions and future research}

We have proposed a new definition of interval-valued restricted equivalence functions with respect to total order considering the width of the intervals and described a construction method for restricted equivalence functions which preserves the widths of intervals. In a similar way, we have presented a construction method for $w$-preserving interval-valued aggregation functions with respect to total orders. Consequently, we have introduced a new definition of a width-based interval-valued similarity measure with respect to total order and proposed a construction method by aggregating restricted equivalence functions. Finally, we have discussed an illustrative example in stereo image matching where the width-based interval-valued similarity measures were used.

The presented experimental study allows us to conclude that considering the width of the intervals in order to measure similarity between intervals is highly beneficial. The proposed method obtains better results, both compared to other interval-valued methods which do not take into account the width of the intervals, and compared to classical methods such as SSD, SAD, NCC, ZNCC or FUZZY. We note that the time complexity of our proposal is the same as the fuzzy method not using intervals $\left(\mathcal{O}\left(N^{2}\right)\right)$ and slightly higher than the classical ones due to the fuzzyfication process.

It is worth mentioning that the use of colour in the extraction of the disparity values is a key point that leads to an improvement in the results. Finally, the presented method can be improved combining filtering steps during the similarity computation and searching for inconsistent disparities in the raw disparity map.

In our future work we are going to study the notions and constructions of interval-valued dissimilarity and interval-valued entropy in line with the presented approach, i.e., with respect to total order and considering the widths of intervals. We also intend to analyze the use of refinement steps to further improve our method.

\section{Acknowledgements}

H. Bustince, C. Marco-Detchart and J. Fernandez were partially supported by Spanish research project TIN2016-77356-P (AEI/FEDER,UE). Z. Takáč has been supported by Project VEGA 1/0614/18. This contribution is also the partial result of the Research \& Development Operational Programme for the project University Scientific Park STU in Bratislava, ITMS 26240220084. C. Wagner and J. M. Garibaldi were partially supported by the UK EPSRC grant EP/P011918/1. 


\section{References}

[1] M.J. Asiáin, H. Bustince, R. Mesiar, A. Kolesárová, Z. Takáč, Negations with respect to admissible orders in the intervalvalued fuzzy set theory, IEEE Transactions on Fuzzy Systems 26 (2018) 556-568.

[2] E. Barrenechea, H. Bustince, B. De Baets, C. Lopez-Molina, Construction of interval-valued fuzzy relations with application to the generation of fuzzy edge images IEEE Transactions on Fuzzy Systems 19 (5) (2011) 819-830.

[3] E. Barrenechea, J. Fernandez, M. Pagola, F. Chiclana, H. Bustince, Construction of interval-valued fuzzy preference relations from ignorance functions and fuzzy preference relations. Application to decision making Knowledge-Based Systems 58 (2014) 33-44.

[4] M. Pagola, Representation of uncertainty by interval valued fuzzy sets. Application to image thresholding, PhD thesis, Universidad Pública de Navarra, 2008

[5] U. Bentkowska, H. Bustince, A. Jurio, M. Pagola, B. Pekala, Decision making with an interval-valued fuzzy preference relation and admissible orders, Applied Soft Computing, 35 (2015) 792-801.

[6] P. Burillo, H. Bustince, Construction theorems for intuitionistic fuzzy sets, Fuzzy Sets and Systems, 84 (1996) $271-281$.

[7] H. Bustince, Indicator of inclusion grade for interval-valued fuzzy sets. Application to approximate reasoning based on interval-valued fuzzy sets, International Journal of Approximate Reasoning 23 (3) (2000) 137-209.

[8] H. Bustince, E. Barrenechea, M. Pagola, Relationship between restricted dissimilarity functions, restricted equivalence functions and normal $E_{N}$-functions: Image thresholding invariant, Pattern Recognition Letters 29(4) (2008) 525-536.

[9] P. Burillo, H. Bustince, Entropy on intuitionistic fuzzy sets and on interval-valued fuzzy sets, Fuzzy Sets and Systems 78 (1996) 305-316.

[10] H. Bustince, E. Barrenechea, M. Pagola, Image thresholding using restricted equivalence functions and maximizing the measure of similarity, Fuzzy Sets and Systems 128(5) (2007) 496-516.

[11] H. Bustince, E. Barrenechea, M. Pagola, Restricted equivalence functions, Fuzzy Sets and Systems 157(17) (2006) 23332346.

[12] H. Bustince, E. Barrenechea, M. Pagola, J. Fernández, Z. Xu, B. Bedregal, J. Montero, H. Hagras, F. Herrera, B. De Baets, A historical account of types of fuzzy sets and their relationship, IEEE Transactions on Fuzzy Systems, 24 (1) (2016) 179-194.

[13] H. Bustince, J. Fernandez, , A. Kolesárová, R. Mesiar, Generation of linear orders for intervals by means of aggregation functions, Fuzzy Sets and Systems 220 (2013) 69-77.

[14] C. Cornelis, G. Deschrijver, E.E. Kerre, Implication in intuitionistic fuzzy and interval-valued fuzzy set theory: construction, classification, application, International Journal of Approximate Reasoning 35 (1) (2004) 55-95.

[15] I. Couso, H. Bustince, From fuzzy sets to interval-valued and Atanassov intuitionistic fuzzy sets: a unified view of different axiomatic measures, IEEE Transactions on Fuzzy Systems, in press.

[16] I. Couso, L. Sánchez, Additive similarity and dissimilarity measures, Fuzzy sets and Systems 322 (2017) 35-53.

[17] H.M. Choi, G.S. Mun, J.Y. Ahn, A medical diagnosis based on interval-valued fuzzy sets, Biomedical EngineeringApplications Basis Communications, 24(4) (2012) 349-354.

[18] P. Couto, A. Jurio, A. Varejao, M. Pagola, H. Bustince, P. Melo-Pinto, An IVFS-based image segmentation methodology for rat gait analysis, Soft Computing, 15(10) (2011) 1937-1944.

[19] G. Deng, L. Song, Y. Jiang and J. Fu, Monotonic Similarity Measures of Interval-Valued Fuzzy Sets and Their Applications, International Journal of Uncertainty, Fuzziness and Knowlege-Based Systems 25 (4) (2017) 515-544.

[20] E. Deza and M.-M. Dezam, Image and Audio Distances, in Dictionary Distances, pages 262-278. Elsevier, 2006.

[21] D. Dubois, H. Prade, Gradualness, uncertainty and bipolarity: Making sense of fuzzy sets, Fuzzy Sets and Systems 192 (2012) 3-24.

[22] O. Faugeras, T. Viéville, E. Theron, J. Vuillemin, B. Hotz, Z. Zhang, L. Moll, P. Bertin, H. Mathieu, P. Fua, G. Berry, and C. Proy. Real-time correlation-based stereo : algorithm, implementations and applications, Research Report RR-2013, INRIA, 1993.

[23] J. Fodor and M. Roubens. Fuzzy preference modelling and multicriteria decision support, in: Theory and Decision Library, Kluwer Academic Publishers, Dordrecht, 1994.

[24] M. Galar, J. Fernandez, G. Beliakov, and H. Bustince. Interval-valued fuzzy sets applied to stereo matching of color images. IEEE Transactions on Image Processing, 20(7):1949-61, 2011.

[25] A. Heidarzade, A new similarity measure for interval type-2 fuzzy sets: Application in fuzzy risk analysis, International Journal of Applied Decision Sciences, 9 (4) (2016) 400-412.

[26] A. Hosni, C. Rhemann, M. Bleyer, C. Rother, and M. Gelautz. Fast Cost-Volume Filtering for Visual Correspondence and Beyond. IEEE Transactions on Pattern Analysis and Machine Intelligence, 35(2):504-511, feb 2013.

[27] L. Hurwicz. The generalised Bayes minimax principle. A criterion for decision making under uncertainty, Cowles Comission Discussion Paper 355, 1951.

[28] A. Jurio, M. Pagola, R. Mesiar, G. Beliakov, H. Bustince, Image magnification using interval information, IEEE Transactions on Image Processing 20 (11) (2011) 3112-3123.

[29] M. Komorníková, R. Mesiar. Aggregation functions on bounded partially ordered sets and their classification. Fuzzy Sets and Systems 175 (1) (2011), 48-56.

[30] X. Liu, Entropy, distance measure and similarity measure of fuzzy sets and their relations. Fuzzy Sets Systems 52 (1992) 305-318.

[31] Z. Lu, and J. Ye, Logarithmic similarity measure between interval-valued fuzzy sets and its fault diagnosis method, Information (Switzerland) 9 (2018) art. no. 36.

[32] R. Mesiar, M. Komorníková, Aggregation Functions on Bounded Posets, in: C. Cornelis et al. (Eds.), 35 Years of Fuzzy Sets Theory (Studies in Fuzziness and Soft Computing, Vol. 261), Springer, Berlin 2010, pp. 3-17. 
[33] G. Nieradka and B. S. Butkiewicz, Features stereo matching based on fuzzy logic, in Proceedings IFSA/EUSFLAT Conference, 2009, pp. 1188-1193.

[34] A. Pradera, G. Beliakov, H. Bustince, B. De Baets, A review of the relationship between implication, negation and aggregation functions from the point of view of material implication, Information Sciences 329 (2016) 357-380.

[35] R. Sambuc. Function phi-flous application a l'aide au diagnostic en pathologie thyroidienne. PhD thesis, University of Marseille, 1975.

[36] J.A. Sanz, A. Fernandez, H. Bustince, F. Herrera, IVTURS: A linguistic fuzzy rule-based classification system based on a new interval-valued fuzzy reasoning method with tuning and rule selection, IEEE Transactions on Fuzzy Systems 21 (3) (2013) 399-411.

[37] D. Scharstein and R. Szeliski. No Title. Internatiopnal Journal of Computer Vision, 47(1/3):7-42, 2002.

[38] C. Sun. Fast Stereo Matching Using Rectangular Subregioning and 3D Maximum-Surface Techniques. Internatiopnal Journal of Computer Vision, 47(1-3):99-117, 2002.

[39] G. Tolt and I. Kalaykov. Measures Based on Fuzzy Similarity for Stereo Matching of Color Images. Soft Computing, 10(12):1117-1126, oct 2006

[40] E. Trucco, V. Roberto, S Tinonin, and M Corbatto. SSD Disparity Estimation for Dynamic Stereo. In BMVC, pages $1-10,1996$

[41] Z.S. Xu, R.R. Yager, Some geometric aggregation operators based on intuitionistic fuzzy sets, International Journal of General Systems 35 (2006) 417-433.

[42] J. Ye, Multicriteria decision-making method based on cosine similarity measures between interval-valued fuzzy sets with risk preference, Economic Computation and Economic Cybernetics Studies and Research 50 (4) (2016) $205-215$.

[43] W. Yu, T. Chen, and J C. Hoe. Real time stereo vision using exponential step cost aggregation on GPU. In Proc. - Int. Conf. Image Process. ICIP, pages 4281-4284, 2009.

[44] R. Zabih and J. Woodfill. Non-parametric local transforms for computing visual correspondence. In Jan-Olof Eklundh, editor, Comput. Vis. - ECCV'94, pages 151-158, Berlin, Heidelberg, 1994. Springer Berlin Heidelberg.

[45] M.Z. Brown, D. Burschka, and G.D. Hager. Advances in computational stereo. IEEE Transactions on Pattern Analysis and Machine Intelligence, 25(8):993-1008, aug 2003. 